\title{
Homology cylinders and the acyclic closure of a free group
}

\author{
TAKUYA SAKASAI
}

\begin{abstract}
We give a Dehn-Nielsen type theorem for the homology cobordism group of homology cylinders by considering its action on the acyclic closure, which was defined by Levine in [12] and [13], of a free group. Then we construct an additive invariant of those homology cylinders which act on the acyclic closure trivially. We also describe some tools to study the automorphism group of the acyclic closure of a free group generalizing those for the automorphism group of a free group or the homology cobordism group of homology cylinders.
\end{abstract}

20F28; 20F34, 57M05, 57M27

\section{Introduction : Dehn-Nielsen's theorem}

Let $\Sigma_{g, 1}(g \geq 0)$ be a compact connected oriented surface of genus $g$ with one boundary component. The fundamental group $\pi_{1} \Sigma_{g, 1}$ of $\Sigma_{g, 1}$ is isomorphic to a free group $F_{2 g}$ of rank $2 g$. We take a word $\zeta \in F_{2 g}$ which corresponds to the boundary loop of $\Sigma_{g, 1}$.

Let $\mathcal{M}_{g, 1}$ be the mapping class group of $\Sigma_{g, 1}$ relative to the boundary. It is the group of all isotopy classes of self-diffeomorphisms of $\Sigma_{g, 1}$ which fix the boundary pointwise. $\mathcal{M}_{g, 1}$ acts on $\pi_{1} \Sigma_{g, 1}$ naturally, so that we have a homomorphism $\sigma: \mathcal{M}_{g, 1} \rightarrow \operatorname{Aut} F_{2 g}$. The following theorem due to Dehn and Nielsen is well known.

Theorem 1.1 (Dehn-Nielsen) The homomorphism $\sigma$ is injective, and its image is

$$
\operatorname{Aut}_{0} F_{2 g}:=\left\{\varphi \in \operatorname{Aut} F_{2 g} \mid \varphi(\zeta)=\zeta\right\} .
$$

From this theorem, we see that an element of $\mathcal{M}_{g, 1}$ is completely characterized by its action on $\pi_{1} \Sigma_{g, 1}$. The action of $\mathcal{M}_{g, 1}$ on $F_{2 g}$ induces that on its nilpotent quotient $N_{k}:=F_{2 g} /\left(\Gamma^{k} F_{2 g}\right)$ for every $k \geq 2$, where $\Gamma^{k} G$ is the $k^{\text {th }}$ term of the lower central series of a group $G$ defined by $\Gamma^{1} G=G$ and $\Gamma^{i} G=\left[\Gamma^{i-1} G, G\right]$ for $i \geq 2$. This defines a homomorphism $\sigma_{k}: \mathcal{M}_{g, 1} \rightarrow \operatorname{Aut} N_{k}$. Note that the restriction of $\sigma_{k}$ to $\operatorname{Ker} \sigma_{k-1}$, whose target is contained in an abelian subgroup of Aut $N_{k}$, is called the 
$(k-2)^{\text {nd }}$ Johnson homomorphism. It has been an important problem to determine its image (see [15]).

Now we consider a generalization of the above argument to the homology cobordism group $\mathcal{H}_{g, 1}$ of homology cylinders. The group $\mathcal{H}_{g, 1}$ has its origin in [5], [3], [14], and it is regarded as an enlargement of $\mathcal{M}_{g, 1}$. One of the main results of this paper is the following Dehn-Nielsen type theorem. $\mathcal{H}_{g, 1}$ has a natural action on the group $F_{2 g}^{\text {acy }}$ called the acyclic closure of $F_{2 g}$, which is a completion of $F_{2 g}$ in a certain sense defined by Levine in [12] and [13], and we will determine the image of the representation $\sigma^{\text {acy }}: \mathcal{H}_{g, 1} \rightarrow \operatorname{Aut} F_{2 g}^{\text {acy }}$ as follows.

Theorem 6.1 The image of $\sigma^{\text {acy }}: \mathcal{H}_{g, 1} \rightarrow$ Aut $F_{2 g}^{\text {acy }}$ is

$$
\operatorname{Aut}_{0} F_{2 g}^{\text {acy }}:=\left\{\varphi \in \operatorname{Aut} F_{2 g}^{\text {acy }} \mid \varphi(\zeta)=\zeta \in F_{2 g}^{\text {acy }}\right\} .
$$

Note that, in this case, the homomorphism $\sigma^{\text {acy }}$ is not injective. Our next result is the construction of a homomorphism

$$
\theta: \operatorname{Ker} \sigma^{\text {acy }} \longrightarrow H_{3}\left(F_{2 g}^{\text {acy }}\right)
$$

which might be able to detect the elements of the kernel of $\sigma^{\text {acy }}$, where the phrase "might be" means that, although we can show that this homomorphism is surjective, it is not known, at present, whether its target is trivial or not. This situation is similar to that of some link concordance invariants defined by Levine [12].

The group Aut $F_{n}^{\text {acy }}$ can be regarded as an enlargement of $\operatorname{Aut} F_{n}$, similar to the situation where $\mathcal{H}_{g, 1}$ enlarges $\mathcal{M}_{g, 1}$, and it embodies a (combinatorial) group-theoretical part of $\mathcal{H}_{g, 1}$ in the case where $n=2 g$. From this, we see that Aut $F_{n}^{\text {acy }}$ itself is an interesting object. We will describe some tools to understand this group - the Johnson homomorphisms with their refinements, and the Magnus representation for $\operatorname{Aut} F_{n}^{\text {acy }}$. They are generalizations of those previously developed by Morita [15] and Kawazumi [9] for Aut $F_{n}$, by Habiro [5], Garoufalidis-Levine [3] and Levine [14] for $\mathcal{H}_{g, 1}$, and by Le Dimet [11] for the Gassner representation of string links.

The author would like to express his gratitude to Professor Shigeyuki Morita for his encouragement and helpful suggestions. The author also would like to thank the referee for useful comments and suggestions.

This research was partially supported by the $21^{\text {st }}$-century COE program at Graduate School of Mathematical Sciences, the University of Tokyo, and by JSPS Research Fellowships for Young Scientists.

Algebraic $6 \mathcal{G}$ Geometric $\mathcal{T}$ opology, Volume 6 (2006) 


\section{Definition of homology cylinders}

We begin by recalling the definition of homology cylinders due to Habiro [5], Garoufalidis-Levine [3] and Levine [14].

A homology cylinder (over $\Sigma_{g, 1}$ ) is a compact oriented 3-manifold $M$ equipped with two embeddings $i_{+}, i_{-}:\left(\Sigma_{g, 1}, p\right) \rightarrow(\partial M, p)$ satisfying that

(1) $i_{+}$is orientation-preserving and $i_{-}$is orientation-reversing,

(2) $\partial M=i_{+}\left(\Sigma_{g, 1}\right) \cup i_{-}\left(\Sigma_{g, 1}\right), i_{+}\left(\Sigma_{g, 1}\right) \cap i_{-}\left(\Sigma_{g, 1}\right)=i_{+}\left(\partial \Sigma_{g, 1}\right)=i_{-}\left(\partial \Sigma_{g, 1}\right)$,

(3) $\left.i_{+}\right|_{\partial \Sigma_{g, 1}}=\left.i_{-}\right|_{\partial \Sigma_{g, 1}}$,

(4) $i_{+}, i_{-}: H_{*}\left(\Sigma_{g, 1}\right) \rightarrow H_{*}(M)$ are isomorphisms,

where $p \in \partial \Sigma_{g, 1}$ is the base point of $\Sigma_{g, 1}$ and $M$. We write a homology cylinder by $\left(M, i_{+}, i_{-}\right)$or simply by $M$.

Example 2.1 $\left(M, i_{+}, i_{-}\right)=\left(\Sigma_{g, 1} \times I, \mathrm{id} \times 1, \varphi \times 0\right)$ gives a homology cylinder for each $\varphi \in \mathcal{M}_{g, 1}$, where collars of $i_{+}\left(\Sigma_{g, 1}\right)$ and $i_{-}\left(\Sigma_{g, 1}\right)$ are stretched half-way along $\partial \Sigma_{g, 1} \times I$.

Two homology cylinders are said to be isomorphic if there exists an orientationpreserving diffeomorphism between the underlying 3-manifolds which is compatible with the embeddings of $\Sigma_{g, 1}$. We denote the set of isomorphism classes of homology cylinders by $\mathcal{C}_{g, 1}$. Given two homology cylinders $M=\left(M, i_{+}, i_{-}\right)$and $N=\left(N, j_{+}, j_{-}\right)$, we can define a new homology cylinder $M \cdot N$ by

$$
M \cdot N=\left(M \cup_{i_{-} \circ\left(j_{+}\right)^{-1}} N, i_{+}, j_{-}\right) .
$$

Then $\mathcal{C}_{g, 1}$ becomes a monoid with the identity element

$$
1_{\mathcal{C}_{g, 1}}:=\left(\Sigma_{g, 1} \times I, \mathrm{id} \times 1, \mathrm{id} \times 0\right) .
$$

This monoid $\mathcal{C}_{g, 1}$ is known as an important object to which the theory of clasper- or clover-surgeries related to finite type invariants of general 3-manifolds is applied.

Instead of the monoid $\mathcal{C}_{g, 1}$, however, we now consider the homology cobordism group $\mathcal{H}_{g, 1}$ of homology cylinders defined as follows. Two homology cylinders $M=$ $\left(M, i_{+}, i_{-}\right)$and $N=\left(N, j_{+}, j_{-}\right)$are homology cobordant if there exists a smooth compact 4-manifold $W$ such that

(1) $\partial W=M \cup(-N) /\left(i_{+}(x)=j_{+}(x), i_{-}(x)=j_{-}(x)\right) \quad x \in \Sigma_{g, 1}$,

(2) the inclusions $M \hookrightarrow W, N \hookrightarrow W$ induce isomorphisms on the homology, 
where $-N$ is $N$ with opposite orientation. Such a manifold $W$ is called a homology cobordism between $M$ and $N$. We denote by $\mathcal{H}_{g, 1}$ the quotient set of $\mathcal{C}_{g, 1}$ with respect to the equivalence relation of homology cobordism. The monoid structure of $\mathcal{C}_{g, 1}$ induces a group structure of $\mathcal{H}_{g, 1}$. In the group $\mathcal{H}_{g, 1}$, the inverse of $\left(M, i_{+}, i_{-}\right)$ is given by $\left(-M, i_{-}, i_{+}\right)$.

The group $\mathcal{H}_{g, 1}$ has the following remarkable properties.

First, $\mathcal{H}_{g, 1}$ contains several groups which relate to the theory of low dimensional topology. As we see in Example 2.1, we can construct a homology cylinder from each element of $\mathcal{M}_{g, 1}$. This correspondence gives an injective monoid homomorphism $\mathcal{M}_{g, 1} \rightarrow \mathcal{C}_{g, 1}$, and moreover, the composite of this homomorphism and the natural projection $\mathcal{C}_{g, 1} \rightarrow \mathcal{H}_{g, 1}$ gives an injective group homomorphism. Therefore $\mathcal{M}_{g, 1}$ is contained in $\mathcal{H}_{g, 1}$. The $g$-component string link concordance group $\mathcal{S}_{g}$ is also contained in $\mathcal{H}_{g, 1}$ [14]. In particular, the (smooth) knot concordance group, which coincides with $\mathcal{S}_{1}$, is contained in $\mathcal{H}_{g, 1}$. Furthermore, we can inject the homology cobordism group $\Theta_{\mathbb{Z}}^{3}$ of homology 3 -spheres into $\mathcal{H}_{g, 1}$ by assigning $M \# 1_{\mathcal{C}_{g, 1}}$ to each homology 3-sphere $M$ up to homology cobordism.

Secondly, we have, as it were, the Milnor-Johnson correspondence, which indicates a similarity between the theory of string links and that of homology cylinders. Hence we can expect that some methods for studying string links (as well as classical knots or links theory in general) are applicable to homology cylinders.

Lastly, we mention about the fundamental group of each homology cylinder. For a given homology cylinder $\left(M, i_{+}, i_{-}\right)$, two homomorphisms $i_{+}, i_{-}: \pi_{1} \Sigma_{g, 1} \rightarrow \pi_{1} M$ are not generally isomorphisms. However, we have the following.

Theorem 2.2 (Stallings [17]) Let $A$ and $B$ be groups and $f: A \rightarrow B$ be a $2-$ connected homomorphism. Then the induced map $f: A /\left(\Gamma^{k} A\right) \longrightarrow B /\left(\Gamma^{k} B\right)$ is an isomorphism for each $k \geq 2$.

Here, a homomorphism $f: A \rightarrow B$ is said to be 2 -connected if $f$ induces an isomorphism on the first homology, and a surjective homomorphism on the second homology. In this paper, the phrase "Stallings' theorem" always means Theorem 2.2. For each homology cylinder $\left(M, i_{+}, i_{-}\right)$, two homomorphisms $i_{+}, i_{-}: F_{2 g} \cong \pi_{1} \Sigma_{g, 1} \rightarrow \pi_{1} M$ are both 2-connected by definition. Hence, they induce isomorphisms on the nilpotent quotients of $F_{2 g}$ and $\pi_{1} M$ by Stallings' theorem. We write $N_{k}$ for $F_{2 g} /\left(\Gamma^{k} F_{2 g}\right)$ as before. For each $k \geq 2$, we define a map $\sigma_{k}: \mathcal{C}_{g, 1} \rightarrow \operatorname{Aut} N_{k}$ by

$$
\sigma_{k}\left(M, i_{+}, i_{-}\right):=\left(i_{+}\right)^{-1} \circ i_{-},
$$


which is seen to be a monoid homomorphism. It can be also checked that $\sigma_{k}\left(M, i_{+}, i_{-}\right)$ depends only on the homology cobordism class of $\left(M, i_{+}, i_{-}\right)$, so that we have a group homomorphism $\sigma_{k}: \mathcal{H}_{g, 1} \rightarrow$ Aut $N_{k}$. Note that the restriction of $\sigma_{k}$ to the subgroup $\mathcal{M}_{g, 1} \subset \mathcal{C}_{g, 1}$ is nothing other than the homomorphism mentioned in Section 1. By definition, the image of the homomorphism $\sigma_{k}$ is contained in

$$
\operatorname{Aut}_{0} N_{k}:=\left\{\begin{array}{l|l}
\varphi \in \operatorname{Aut} N_{k} & \begin{array}{c}
\text { There exists a lift } \tilde{\varphi} \in \operatorname{End} F_{2 g} \text { of } \varphi \\
\text { satisfying } \tilde{\varphi}(\zeta) \equiv \zeta \bmod \Gamma^{k+1} F_{2 g} .
\end{array}
\end{array}\right\} .
$$

On the other hand, Garoufalidis-Levine and Habegger independently showed the following.

Theorem 2.3 (Garoufalidis-Levine [3], Habegger [4]) For $k \geq 2$, Image $\sigma_{k}=$ $\operatorname{Aut}_{0} N_{k}$

Note that the $(k-2)^{\text {nd }}$ Johnson homomorphism is obtained by restricting $\sigma_{k}$ to $\operatorname{Ker} \sigma_{k-1}$. From this theorem, we can determine its image completely (see [3]).

Now we have the following question. Recall that in the case of the mapping class group, $\sigma_{k}: \mathcal{M}_{g, 1} \rightarrow$ Aut $N_{k}$ are induced from a single homomorphism $\sigma: \mathcal{M}_{g, 1} \rightarrow \operatorname{Aut} F_{2 g}$. Then our question is:

Question 2.4 Does there exist a homomorphism $\mathcal{H}_{g, 1} \rightarrow$ Aut $G$ for some group $G$ which induces $\sigma_{k}: \mathcal{H}_{g, 1} \rightarrow$ Aut $N_{k}$ for all $k \geq 2$ ?

Some answers to it are given in Remark 2.3 in [3]. Namely, we have a homomorphism $\sigma^{\text {nil: }} \mathcal{H}_{g, 1} \rightarrow \operatorname{Aut} F_{2 g}^{\text {nil }}$ by combining the homomorphisms $\sigma_{k}$ for all $k \geq 2$, where $F_{2 g}^{\text {nil }}:=\lim _{\leftarrow} N_{k}$ is the nilpotent completion of $F_{2 g}$. However, $F_{2 g}^{\text {nil }}$ is too big to treat. Then, the usage of the residually nilpotent algebraic closure of $F_{2 g}$, which is a countable (as a set) subgroup of $F_{2 g}^{\text {nil }}$, is also suggested. However, as commented there, we do not know whether its second homology is trivial or not. The vanishing of it is efficiently used in several situations. In this paper, we suggest the usage of the acyclic closure (or HE-closure) $F_{2 g}^{\text {acy }}$ of $F_{2 g}$ to overcome them through the argument in subsequent sections.

\section{Construction of an enlargement of $\operatorname{Aut} F_{n}$}

Now we fix an integer $n \geq 0$. In this section, we define a group, denoted by $\mathcal{B}_{n}$, which can be regarded as an enlargement of Aut $F_{n}$. The construction of this group is analogous to that of the group $\mathcal{H}_{g, 1}$ of homology cylinders. We consider all the 
arguments in a group level. We first construct a monoid $\mathcal{A}_{n}$, which enlarges the group Aut $F_{n}$, and then we obtain the group $\mathcal{B}_{n}$ by taking the quotient of $\mathcal{A}_{n}$ by certain equivalence relation.

Step 1 The construction of the monoid $\mathcal{A}_{n}$ proceeds as follows. Let $\mathcal{A}_{n}$ be the set of all equivalence classes of triplets $\left(G, \varphi_{+}, \varphi_{-}\right)$consisting of a finitely presentable group $G$ and 2-connected homomorphisms $\varphi_{+}, \varphi_{-}: F_{n} \rightarrow G$, where two triplets $\left(G, \varphi_{+}, \varphi_{-}\right),\left(G^{\prime}, \psi_{+}, \psi_{-}\right)$are said to be equivalent if there exists an isomorphism $\rho: G \stackrel{\cong}{\longrightarrow} G^{\prime}$ which makes the following diagram commutative:

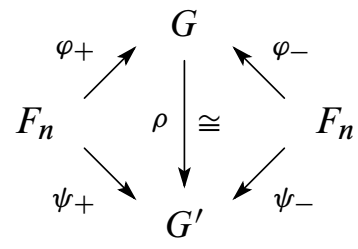

Note that for such a triplet $\left(G, \varphi_{+}, \varphi_{-}\right)$, homomorphisms $\varphi_{+}$and $\varphi_{-}$are injective, which follows from Stallings' theorem and the fact that $F_{n}$ is residually nilpotent.

We define a multiplication $\cdot$ on $\mathcal{A}_{n}$ as follows:

$$
\begin{array}{ccc}
\mathcal{A}_{n} \times \mathcal{A}_{n} & \rightarrow & \mathcal{A}_{n} \\
\Psi & & \Psi \\
\left(\left(G, \varphi_{+}, \varphi_{-}\right),\left(G^{\prime}, \psi_{+}, \psi_{-}\right)\right) & \mapsto & \left(G * F_{n} G^{\prime}, \varphi_{+}, \psi_{-}\right)
\end{array}
$$

where $G * F_{n} G^{\prime}$ is obtained by taking the amalgamated product of $G$ and $G^{\prime}$ with respect to $\varphi_{-}: F_{n} \rightarrow G$ and $\psi_{+}: F_{n} \rightarrow G^{\prime}$. Since $\varphi_{-}$and $\psi_{+}$are both injective, we can use the Mayer-Vietoris exact sequence for the homology of amalgamated products (see [1]), so that the above map gives a well-defined monoid structure of $\mathcal{A}_{n}$ with the identity element $\left(F_{n}\right.$, id, id $)$.

Example 3.1 Aut $F_{n}$ can be seen as a submonoid of $\mathcal{A}_{n}$ by assigning to each automorphism $\varphi$ of $F_{n}$ an element $\left(F_{n}\right.$, id, $\left.\varphi\right)$ of $\mathcal{A}_{n}$. This correspondence gives an injective monoid homomorphism as shown in Corollary 5.2.

Example 3.2 Consider the monoid of all 2-connected endomorphisms of $F_{n}$. To each 2-connected endomorphism $\varphi$ of $F_{n}$, we can assign $\left(F_{n}\right.$, id, $\left.\varphi\right) \in \mathcal{A}_{n}$. This correspondence is also an injective monoid homomorphism. For example, consider an endomorphism $\psi: F_{2}=\left\langle x_{1}, x_{2}\right\rangle \rightarrow F_{2}$ defined by

$$
\psi\left(x_{1}\right)=x_{1} x_{2} x_{1} x_{2}^{-1} x_{1}^{-1}, \quad \psi\left(x_{2}\right)=x_{2}
$$


where we take $n=2$. As we will see in Example 5.15, $\psi$ is not an automorphism of $F_{2}$ but a 2-connected endomorphism. Hence $\left(F_{2}\right.$, id, $\left.\psi\right)$ gives an example of elements of $\mathcal{A}_{2}$ which are not contained in $\operatorname{Aut} F_{2}$.

Example 3.3 For each homology cylinder $\left(M, i_{+}, i_{-}\right)$, we can obtain an element $\left(\pi_{1} M, i_{+}, i_{-}\right)$of $\mathcal{A}_{2 g}$. This correspondence gives a monoid homomorphism $\mathcal{C}_{g, 1} \rightarrow$ $\mathcal{A}_{2 g}$.

Step 2 We construct the group $\mathcal{B}_{n}$ from the monoid $\mathcal{A}_{n}$ as follows. Two elements $\left(G, \varphi_{+}, \varphi_{-}\right),\left(G^{\prime}, \psi_{+}, \psi_{-}\right)$of $\mathcal{A}_{n}$ are said to be homology cobordant if there exist a finitely presentable group $\widetilde{G}$ and 2-connected homomorphisms

$$
\varphi: G \longrightarrow \widetilde{G}, \quad \psi: G^{\prime} \longrightarrow \widetilde{G}
$$

which make the following diagram commutative:

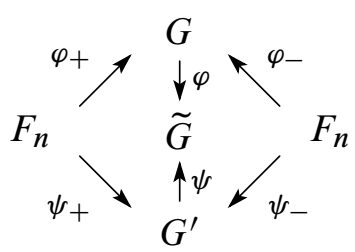

We define $\mathcal{B}_{n}$ to be the quotient set of $\mathcal{A}_{n}$ with respect to the equivalence relation generated by the relation of homology cobordism. Then we can endow with a group structure on $\mathcal{B}_{n}$ from the monoid structure of $\mathcal{A}_{n}$. In $\mathcal{B}_{n}$, the inverse element of $\left(G, \varphi_{+}, \varphi_{-}\right)$is given by $\left(G, \varphi_{-}, \varphi_{+}\right)$. Indeed, $G * F_{n} G \stackrel{\operatorname{id} *_{F_{n}} \text { id }}{\longrightarrow} G \stackrel{\varphi_{+}}{\longleftarrow} F_{n}$ gives a homology cobordism between $\left(G * F_{n} G, \varphi_{+}, \varphi_{+}\right)=\left(G, \varphi_{+}, \varphi_{-}\right) \cdot\left(G, \varphi_{-}, \varphi_{+}\right)$and $\left(F_{n}\right.$, id, id $)$.

Example 3.4 The monoid homomorphism $\mathcal{C}_{g, 1} \rightarrow \mathcal{A}_{2 g}$ considered in Example 3.3 induces a group homomorphism $\mathcal{H}_{g, 1} \rightarrow \mathcal{B}_{2 g}$. This homomorphism gives an enlargement of the inclusion $\sigma: \mathcal{M}_{g, 1} \rightarrow \operatorname{Aut} F_{2 g}$.

Fundamental properties of the group $\mathcal{B}_{n}$ will be mentioned in Section 5 after seeing a relationship with the acyclic closure of a free group.

\section{The acyclic closure of a group}

The concept of the acyclic closure (or HE-closure in [13]) of a group was defined as a variation of the algebraic closure of a group by Levine in [12], [13]. We briefly 
summarize the definition and fundamental properties. We also refer to Hillman's book [7]. The proofs of the propositions in this section are almost the same as those for the algebraic closure in [12] (see Remark 4.9).

Definition 4.1 Let $G$ be a group, and let $F_{n}=\left\langle x_{1}, x_{2}, \ldots, x_{n}\right\rangle$ be a free group of rank $n$.

(i) We call each element $w=w\left(x_{1}, x_{2}, \ldots, x_{n}\right)$ of $G * F_{n}$ a monomial. A monomial $w$ is said to be acyclic if

$$
w \in \operatorname{Ker}\left(G * F_{n} \stackrel{\text { proj }}{\longrightarrow} F_{n} \longrightarrow H_{1}\left(F_{n}\right)\right) .
$$

(ii) Consider the following "equation" with variables $x_{1}, x_{2}, \ldots, x_{n}$ :

$$
\left\{\begin{array}{rl}
x_{1} & =w_{1}\left(x_{1}, x_{2}, \ldots, x_{n}\right) \\
x_{2} & =w_{2}\left(x_{1}, x_{2}, \ldots, x_{n}\right) \\
& \vdots \\
x_{n} & =w_{n}\left(x_{1}, x_{2}, \ldots, x_{n}\right)
\end{array} .\right.
$$

When all monomials $w_{1}, w_{2}, \ldots, w_{n}$ are acyclic, we call such an equation an acyclic system over $G$. (iii) A group $G$ is said to be acyclically closed if every acyclic system over $G$ with $n$ variables has a unique solution in $G$ for any $n \geq 0$.

We denote the phrase "acyclically closed" by AC, for short.

Example 4.2 Let $G$ be an abelian group. For $g_{1}, g_{2}, g_{3} \in G$, consider the equation

$$
\left\{\begin{array}{l}
x_{1}=g_{1} x_{1} g_{2} x_{2} x_{1}^{-1} x_{2}^{-1} \\
x_{2}=x_{1} g_{3} x_{1}^{-1}
\end{array},\right.
$$

which is an acyclic system. Then we have a unique solution $x_{1}=g_{1} g_{2}, x_{2}=g_{3}$.

As we can expect from this example, all abelian groups are AC. In fact, all nilpotent groups and the nilpotent completion of a group are $\mathrm{AC}$, which are deduced from the following fundamental properties of $\mathrm{AC}$-groups and the fact that the trivial group is AC.

Proposition 4.3 (Proposition 1 in [12]) (a) Let $\left\{G_{\alpha}\right\}$ be a family of $A C$-subgroups of an AC-group $G$. Then $\bigcap_{\alpha} G_{\alpha}$ is also an AC-subgroup of $G$.

(b) Let $\left\{G_{\alpha}\right\}$ be a family of $A C$-groups. Then $\prod_{\alpha} G_{\alpha}$ is also an AC-group.

(c) When $G$ is a central extension of $H$, then $G$ is an AC-group if and only if $H$ is an AC-group. 
(d) For each direct system (resp. inverse system) of AC-groups, the direct limit (resp. inverse limit) is also an $A C$-group.

Next we define the acyclic closure of a group.

Proposition 4.4 (Proposition 3 in [12]) For any group $G$, there exists a pair of a group $G^{\text {acy }}$ and a homomorphism $\iota_{G}: G \rightarrow G^{\text {acy }}$ satisfying the following properties:

(1) $G^{\text {acy }}$ is an AC-group.

(2) Let $f: G \rightarrow A$ be a homomorphism and suppose that $A$ is an AC-group. Then there exists a unique homomorphism $f^{\text {acy: }} G^{\text {acy }} \rightarrow A$ which satisfies $f^{\text {acy }} \circ \iota_{G}=f$.

Moreover such a pair is unique up to isomorphisms.

Definition 4.5 We call $\iota_{G}$ (or $G^{\text {acy }}$ ) obtained above the acyclic closure of $G$.

Taking the acyclic closure of a group is functorial, namely, for each group homomorphism $f: G_{1} \rightarrow G_{2}$, we obtain a homomorphism $f^{\text {acy: }} G_{1}^{\text {acy }} \rightarrow G_{2}^{\text {acy }}$ by applying the universal property of $G_{1}^{\text {acy }}$ to the homomorphism $\iota_{G_{2}} \circ f$, and the composition of homomorphisms induces that of the corresponding homomorphisms on acyclic closures.

The most important properties of the acyclic closure are the following.

Proposition 4.6 (Proposition 4 in [12]) For every group $G$, the acyclic closure $\iota_{G}: G \rightarrow G^{\text {acy }}$ is 2-connected.

Proposition 4.7 (Proposition 5 in [12]) Let $G$ be a finitely generated group and $H$ be a finitely presentable group. For each 2-connected homomorphism $f: G \rightarrow H$, the induced homomorphism $f^{\text {acy }}: G^{\text {acy }} \rightarrow H^{\text {acy }}$ on acyclic closures is an isomorphism.

From Proposition 4.6 and Stallings' theorem, the nilpotent quotients of a group and those of its acyclic closure are isomorphic. Note that the homomorphism $\iota_{G}$ is not necessarily injective.

Proposition 4.8 (Proposition 6 in [12]) For any finitely presentable group $G$, there exists a sequence of finitely presentable groups and homomorphisms

$$
G=P_{0} \rightarrow P_{1} \rightarrow P_{2} \rightarrow \cdots \rightarrow P_{k} \rightarrow P_{k+1} \rightarrow \cdots
$$

satisfying the following properties: 
(1) $G^{\text {acy }}=\lim _{\longrightarrow} P_{k}$, and $\iota_{G}: G \rightarrow G^{\text {acy }}$ coincides with the limit map of the above sequence.

(2) $G \rightarrow P_{k}$ is a 2-connected homomorphism.

From this proposition, we see, in particular, that the acyclic closure of a finitely presentable group is a countable set.

Remark 4.9 Here, we comment on the proofs of the above propositions. In the argument of the algebraic closure in [12], Levine used the condition that a group $H$ is finitely normally generated by a subgroup $G$. In the case of the acyclic closure, we need the following alternative condition: the group $H$ is said to be finitely homologically generated by a subgroup $G$ if

(1) The inclusion $G \rightarrow H$ induces a surjective homomorphism $H_{1}(G) \rightarrow H_{1}(H)$.

(2) $H$ is generated by $G$ together with finite elements of $H$.

As for the invisible subgroup, we need not change its definition.

\section{Structures of the groups $\mathcal{B}_{n}$ and $A u t F_{n}^{\text {acy }}$}

Using the results in the last section, we consider the acyclic closure $\iota_{F_{n}}: F_{n} \rightarrow F_{n}^{\text {acy }}$ of $F_{n}$. Since the nilpotent completion $F_{n}^{\text {nil }}$ of $F_{n}$ is AC, there exists a unique homomorphism $p: F_{n}^{\text {acy }} \rightarrow F_{n}^{\text {nil }}$ such that $p \circ \iota^{\imath} F_{n}$ coincides with the natural map $F_{n} \rightarrow F_{n}^{\text {nil }}$, which is known to be injective. Hence $\iota_{F_{n}}$ is also injective.

For each element $\left(G, \varphi_{+}, \varphi_{-}\right)$of $\mathcal{A}_{n}$, we have a commutative diagram

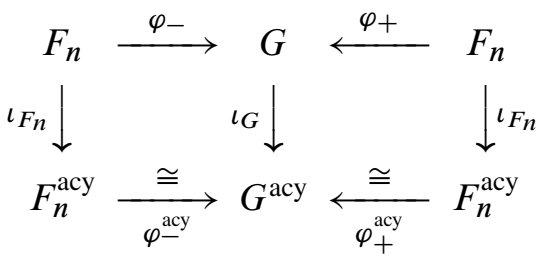

by Proposition 4.7. From this, we obtain a monoid homomorphism defined by

$$
\Phi: \mathcal{A}_{n} \longrightarrow \operatorname{Aut} F_{n}^{\text {acy }} \quad\left(\left(G, \varphi_{+}, \varphi_{-}\right) \mapsto\left(\varphi_{+}^{\text {acy }}\right)^{-1} \circ \varphi_{-}^{\text {acy }}\right)
$$

and it induces a group homomorphism $\Phi: \mathcal{B}_{n} \rightarrow$ Aut $F_{n}^{\text {acy }}$ by the commutativity of the diagram $(*)$ in Section 3 whose homomorphisms are all 2-connected. 
Theorem 5.1 For each $n \geq 0$, the homomorphism $\Phi: \mathcal{B}_{n} \rightarrow$ Aut $F_{n}^{\text {acy }}$ is an isomorphism.

Proof Assume that $\left(G, \varphi_{+}, \varphi_{-}\right) \in \operatorname{Ker} \Phi$. Then $\varphi_{+}^{\text {acy }}=\varphi_{-}^{\text {acy }}: F_{n}^{\text {acy }} \rightarrow G^{\text {acy }}$, so that we have $\iota_{G} \circ \varphi_{+}=\varphi_{+}^{\text {acy }} \circ \iota_{F_{n}}=\varphi_{-}^{\text {acy }} \circ \iota_{F_{n}}=\iota_{G} \circ \varphi_{-}$. By Proposition 4.8, for large $k \geq 0$, we have $i_{k} \circ \varphi_{+}=i_{k} \circ \varphi_{-}: F_{n} \rightarrow P_{k}$ where $P_{k}$ is the $k^{\text {th }}$ group of a sequence whose direct limit gives $G^{\text {acy }}$, and $i_{k}: G \rightarrow P_{k}$ is the composite of homomorphisms of the sequence from $G=P_{0}$ up to $P_{k}$. When we write $\varphi:=i_{k} \circ \varphi_{+}=i_{k} \circ \varphi_{-}: F_{n} \rightarrow P_{k}$, then $\left(G, \varphi_{+}, \varphi_{-}\right) \in \mathcal{A}_{n}$ is homology cobordant to the identity element $\left(F_{n}\right.$, id, id $)$ by a homology cobordism $G \stackrel{i_{k}}{\longrightarrow} P_{k} \stackrel{\varphi}{\leftarrow} F_{n}$. This shows that $\Phi$ is injective.

On the other hand, given $\varphi \in \operatorname{Aut} F_{n}^{\text {acy }}$, we set $f:=\varphi \circ \iota_{F_{n}}: F_{n} \rightarrow F_{n}^{\text {acy }}$. By Proposition 4.8, we have a sequence $\left\{P_{k}\right\}$ of finitely presentable groups whose direct limit is $F_{n}^{\text {acy }}$. For large $k \geq 0$, we can take a lift $\tilde{f}: F_{n} \rightarrow P_{k}$ of $f$ with respect to the limit map $\iota: P_{k} \rightarrow F_{n}^{\text {acy }}$, that is, we have $\iota \circ \tilde{f}=f$. By definition, we have $\iota \circ i_{k}=\iota_{F_{n}}$ where $i_{k}: F_{n} \rightarrow P_{k}$ is the composite of homomorphisms in the sequence. We can see that $i_{k}$ and $\tilde{f}$ are 2-connected homomorphisms, so that $\left(P_{k}, i_{k}, \widetilde{f}\right)$ defines an element of $\mathcal{A}_{n}$. Taking their acyclic closures, we obtain $\Phi\left(P_{k}, i_{k}, \tilde{f}\right)=\varphi$. This completes the proof.

Corollary 5.2 The monoid homomorphism Aut $F_{n} \rightarrow \mathcal{A}_{n}$ and the group homomorphism Aut $F_{n} \rightarrow \mathcal{B}_{n} \cong$ Aut $F_{n}^{\text {acy }}$ described in Section 3 are both injective.

Proof By the universal property of $F_{n}^{\text {acy }}$, two automorphisms of $F_{n}^{\text {acy }}$ are the same if and only if they coincide on the subgroup $F_{n} \subset F_{n}^{\text {acy }}$. The claim follows from this.

Hereafter we identify $\mathcal{B}_{n}$ with $\operatorname{Aut} F_{n}^{\text {acy }}$, and use only the latter. In the rest of this section, we describe some fundamental tools for understanding the structure of the group Aut $F_{n}^{\text {acy }}$.

The Johnson homomorphisms By Stallings' theorem, the inclusion $F_{n} \hookrightarrow F_{n}^{\text {acy }}$ induces isomorphisms on their nilpotent quotients. Therefore we have a natural homomorphism $\Phi_{k}:$ Aut $F_{n}^{\text {acy }} \longrightarrow$ Aut $N_{k}$ for each $k \geq 2$.

Proposition 5.3 For all $k \geq 2$, the homomorphisms $\Phi_{k}$ : Aut $F_{n}^{\text {acy }} \rightarrow$ Aut $N_{k}$ are surjective.

Proof Given an element $f \in \operatorname{Aut} N_{\underline{k}}$, we denote by $\tilde{f} \in \operatorname{End} F_{n}$ a lift of $f$. Since $\tilde{f}$ induces an automorphism on $N_{2}, \tilde{f}$ is a 2-connected endomorphism. Then $\tilde{f}^{\text {acy }} \in$ Aut $F_{n}^{\text {acy }}$ is induced and it satisfies $\Phi_{k}\left(\tilde{f}^{\text {acy }}\right)=f$. 
By using $\Phi_{k}$, the Johnson homomorphism is defined as follows (see also [15] and [9]). We define a filtration of Aut $F_{n}^{\text {acy }}$ by

$$
\operatorname{Aut} F_{n}^{\text {acy }}[1]:=\operatorname{Aut} F_{n}^{\text {acy }}, \quad \operatorname{Aut} F_{n}^{\text {acy }}[k]:=\operatorname{Ker} \Phi_{k} \quad(k \geq 2) .
$$

On the other hand, we have an exact sequence

$$
0 \longrightarrow \operatorname{Hom}\left(H_{1}\left(F_{n}\right),\left(\Gamma^{k} F_{n}\right) /\left(\Gamma^{k+1} F_{n}\right)\right) \longrightarrow \operatorname{Aut} N_{k+1} \longrightarrow \operatorname{Aut} N_{k} \longrightarrow 1,
$$

where $\left(\Gamma^{k} F_{n}\right) /\left(\Gamma^{k+1} F_{n}\right)$ is known to be isomorphic to the degree $k$ part of the graded Lie algebra (over $\mathbb{Z}$ ) freely generated by the elements of $H_{1}\left(F_{n}\right)$, so that Aut $N_{2}$ acts on it. Explicitly, the isomorphism

$$
\begin{aligned}
\operatorname{Ker}\left(\operatorname{Aut} N_{k+1} \rightarrow \operatorname{Aut} N_{k}\right) \stackrel{\sim}{\longrightarrow} & \operatorname{Hom}\left(H_{1}\left(F_{n}\right),\left(\Gamma^{k} F_{n}\right) /\left(\Gamma^{k+1} F_{n}\right)\right) \\
& =\operatorname{Hom}\left(F_{n},\left(\Gamma^{k} F_{n}\right) /\left(\Gamma^{k+1} F_{n}\right)\right)
\end{aligned}
$$

is given by assigning to $f \in \operatorname{Ker}\left(\operatorname{Aut} N_{k+1} \rightarrow \operatorname{Aut} N_{k}\right)$ the homomorphism

$$
F_{n} \ni x_{i} \mapsto \tilde{f}\left(x_{i}\right) x_{i}^{-1} \in\left(\Gamma^{k} F_{n}\right) /\left(\Gamma^{k+1} F_{n}\right)
$$

where $\tilde{f} \in \operatorname{End} F_{n}$ is a lift of $f$ and $\left\langle x_{1}, \ldots, x_{n}\right\rangle$ is a generating system of $F_{n}$. Note that this expression does not depend on the choices involved. If we define $J_{k}:=\left.\Phi_{k+1}\right|_{\text {Aut } F_{n}^{\text {acy }}[k]}$, we obtain an exact sequence

$$
1 \rightarrow \operatorname{Aut} F_{n}^{\text {acy }}[k+1] \longrightarrow \operatorname{Aut} F_{n}^{\text {acy }}[k] \stackrel{J_{k}}{\longrightarrow} \operatorname{Hom}\left(H_{1}\left(F_{n}\right),\left(\Gamma^{k} F_{n}\right) /\left(\Gamma^{k+1} F_{n}\right)\right) \rightarrow 1 .
$$

We call the homomorphism $J_{k}$ the $(k-1)^{\text {st }}$ Johnson homomorphism. Note that $J_{k}$ is Aut $F_{n}^{\text {acy }}$-equivariant, where Aut $F_{n}^{\text {acy }}$ acts on $\operatorname{Aut} F_{n}^{\text {acy }}[k]$ by conjugation and acts on the target through $\Phi_{2}$.

Example 5.4 Consider the 2-connected endomorphism $\psi$ in Example 3.2. As an element of Aut $F_{2}^{\text {acy }}, \psi$ belongs to Aut $F_{2}^{\text {acy }}[2]$. We calculate the image by the first Johnson homomorphism $J_{2}$. We write $H:=H_{1}\left(F_{2}\right)$ and consider isomorphisms

$$
\operatorname{Hom}\left(H_{1}\left(F_{2}\right),\left(\Gamma^{2} F_{2}\right) /\left(\Gamma^{3} F_{2}\right)\right) \cong H^{*} \otimes\left(\Gamma^{2} F_{2}\right) /\left(\Gamma^{3} F_{2}\right) \cong H^{*} \otimes\left(\wedge^{2} H\right) .
$$

Then we have $J_{2}(\psi)=x_{1}^{*} \otimes\left(x_{2} \wedge x_{1}\right)$.

A refinement of the Johnson homomorphisms For each $k \geq 2$, we now give a refinement of the Johnson homomorphism whose target is abelian and bigger than that of the original. To construct the refinement, we need to fix a generating system $\left\langle x_{1}, \ldots, x_{n}\right\rangle$ of $F_{n}$. We show the following. 
Theorem 5.5 For each $k \geq 2$, the Johnson homomorphism $J_{k}$ has a refinement

$$
\widetilde{J}_{k}: \operatorname{Aut} F_{n}^{\text {acy }}[k] \longrightarrow \operatorname{Hom}\left(H_{1}\left(F_{n}\right),\left(\Gamma^{k} F_{n}\right) /\left(\Gamma^{2 k-1} F_{n}\right)\right)
$$

whose target is also a finitely generated free abelian group. In fact, the composite with the natural projection $p_{1}:\left(\Gamma^{k} F_{n}\right) /\left(\Gamma^{2 k-1} F_{n}\right) \rightarrow\left(\Gamma^{k} F_{n}\right) /\left(\Gamma^{k+1} F_{n}\right)$ is the original Johnson homomorphism $J_{k}$. Moreover $\widetilde{J}_{k}$ is surjective, and the kernel of $\widetilde{J}_{k}$ coincides with Aut $F_{n}^{\text {acy }}[2 k-1]$, so that we have an exact sequence

$$
1 \rightarrow \operatorname{Aut} F_{n}^{\text {acy }}[2 k-1] \rightarrow \operatorname{Aut} F_{n}^{\text {acy }}[k] \stackrel{\widetilde{J}_{k}}{\longrightarrow} \operatorname{Hom}\left(H_{1}\left(F_{n}\right),\left(\Gamma^{k} F_{n}\right) /\left(\Gamma^{2 k-1} F_{n}\right)\right) \rightarrow 1 .
$$

Note that for each $k \geq 2$, we have a direct sum decomposition

$$
\left(\Gamma^{k} F_{n}\right) /\left(\Gamma^{2 k-1} F_{n}\right) \cong \bigoplus_{j=k}^{2 k-2}\left(\left(\Gamma^{j} F_{n}\right) /\left(\Gamma^{j+1} F_{n}\right)\right)
$$

which is given by iterated extensions of $\left(\Gamma^{k} F_{n}\right) /\left(\Gamma^{k+1} F_{n}\right)$ by finitely generated free abelian groups $\left(\Gamma^{i} F_{n}\right) /\left(\Gamma^{i+1} F_{n}\right)$ for $k+1 \leq i \leq 2 k-2$. Therefore $\left(\Gamma^{k} F_{n}\right) /\left(\Gamma^{2 k-1} F_{n}\right)$ is also a finitely generated free abelian group. We also note that this direct sum decomposition is not canonical, except for the first projection $p_{1}:\left(\Gamma^{k} F_{n}\right) /\left(\Gamma^{2 k-1} F_{n}\right) \rightarrow\left(\Gamma^{k} F_{n}\right) /\left(\Gamma^{k+1} F_{n}\right)$.

The proof of Theorem 5.5 essentially uses the following.

Lemma 5.6 If we set Aut $F_{n}[k]:=\operatorname{Ker}\left(\operatorname{Aut} F_{n} \rightarrow \operatorname{Aut} N_{k}\right)$, then

$$
\begin{array}{rlrl}
\tilde{J}_{k}: \operatorname{Aut} F_{n}[k] & \rightarrow \operatorname{Hom}\left(F_{n},\left(\Gamma^{k} F_{n}\right) /\left(\Gamma^{2 k-1} F_{n}\right)\right) \\
\Psi & & \Psi \\
\varphi & \mapsto \quad\left(x_{i} \mapsto \varphi\left(x_{i}\right) x_{i}^{-1}\right)
\end{array}
$$

is a well-defined homomorphism.

Proof Given $\varphi, \psi \in \operatorname{Aut} F_{n}[k]$, we have

$$
\tilde{J}_{k}(\varphi \psi)\left(x_{i}\right)=\varphi\left(\psi\left(x_{i}\right)\right) x_{i}^{-1}=\varphi\left(\psi\left(x_{i}\right) x_{i}^{-1}\right) \cdot \varphi\left(x_{i}\right) x_{i}^{-1} .
$$

Since $\varphi\left(x_{i}\right) x_{i}^{-1}, \psi\left(x_{i}\right) x_{i}^{-1} \in \Gamma^{k} F_{n}$, and $\left(\Gamma^{k} F_{n}\right) /\left(\Gamma^{2 k-1} F_{n}\right)$ is abelian, it suffices to show that Aut $F_{n}[k]$ acts on $\left(\Gamma^{k} F_{n}\right) /\left(\Gamma^{2 k-1} F_{n}\right)$ trivially. Every element $g \in$ $\left(\Gamma^{k} F_{n}\right) /\left(\Gamma^{2 k-1} F_{n}\right)$ can be written in a form $\left.\left.g=\prod_{i=1}^{l}\left[\cdots\left[g_{i 1}, g_{i 2}\right], g_{i 3}\right], \cdots\right], g_{i k}\right]$ where $g_{i j} \in F_{n}$, so that it suffices to show our claim in the case of $\left.\left.g=\left[\cdots\left[g_{1}, g_{2}\right], g_{3}\right], \cdots\right], g_{k}\right]$ where $g_{i} \in F_{n}$.

Since $\varphi \in \operatorname{Aut} F_{n}[k]$, we see $\left.\left.\varphi(g)=\left[\cdots\left[g_{1} r_{1}, g_{2} r_{2}\right], g_{3} r_{3}\right], \cdots\right], g_{k} r_{k}\right]$ for some $r_{i} \in$ $\Gamma^{k} F_{n}$. We write $\left.\left.g^{(l)}:=\left[\cdots\left[g_{1}, g_{2}\right], g_{3}\right], \cdots\right], g_{l}\right] \in \Gamma^{l} F_{n}$ for $2 \leq l \leq k$. Now we 
show that $g^{(l)} \equiv \varphi\left(g^{(l)}\right)\left(\bmod \Gamma^{k+l-1} F_{n}\right)$ by the induction on $l$. Our claim follows from it. For $l=2$,

$$
\begin{aligned}
\varphi\left(g^{(2)}\right) & =\left[g_{1} r_{1}, g_{2} r_{2}\right] \\
& =\left[g_{1} r_{1}, g_{2}\right] \cdot{ }^{g_{2}}\left[g_{1} r_{1}, r_{2}\right] \\
& ={ }^{g_{1}}\left[r_{1}, g_{2}\right] \cdot\left[g_{1}, g_{2}\right] \cdot{ }^{g_{2}}\left[g_{1} r_{1}, r_{2}\right] \\
& \equiv g^{(2)} \quad\left(\bmod \Gamma^{k+1} F_{n}\right)
\end{aligned}
$$

where we write ${ }^{a}[b, c]$ for $a[b, c] a^{-1}$. When $g^{(i)} \equiv \varphi\left(g^{(i)}\right) \quad\left(\bmod \Gamma^{k+i-1} F_{n}\right)$ follows for $2 \leq i \leq l$, we see

$$
\begin{aligned}
\varphi\left(g^{(l+1)}\right) & =\left[\varphi\left(g^{(l)}\right), g_{l+1} r_{l+1}\right] \\
& =\left[\varphi\left(g^{(l)}\right), g_{l+1}\right] \cdot g^{g_{+1}}\left[\varphi\left(g^{(l)}\right), r_{l+1}\right] \\
& =\left[g^{(l)} r, g_{l+1}\right] \cdot g_{l+1}\left[\varphi\left(g^{(l)}\right), r_{l+1}\right] \quad \text { for some } r \in \Gamma^{k+l-1} \\
& ={ }^{(l)}\left[r, g_{l+1}\right] \cdot\left[g^{(l)}, g_{l+1}\right] \cdot g^{g+1}\left[\varphi\left(g^{(l)}\right), r_{l+1}\right] \\
& \equiv g^{(l+1)} \quad\left(\bmod \Gamma^{k+l} F_{n}\right)
\end{aligned}
$$

and this completes the proof.

By Lemma 5.6, we see that $\widetilde{J}_{k}$ gives a refinement of the Johnson homomorphism for Aut $F_{n}$.

Proof of Theorem 5.5 If we restrict $\Phi_{2 k-1}:$ Aut $F_{n}^{\text {acy }} \rightarrow$ Aut $N_{2 k-1}$ to the subgroup Aut $F_{n}^{\text {acy }}[k]$, its image is contained in $\operatorname{Ker}\left(\operatorname{Aut} N_{2 k-1} \rightarrow \operatorname{Aut} N_{k}\right)$. On the other hand, the map

$$
\begin{array}{ccc}
\bar{J}_{k}: \operatorname{Ker}\left(\operatorname{Aut} N_{2 k-1} \rightarrow \operatorname{Aut} N_{k}\right) & \rightarrow \operatorname{Hom}\left(F_{n},\left(\Gamma^{k} F_{n}\right) /\left(\Gamma^{2 k-1} F_{n}\right)\right) \\
\Psi & & \cup \\
f & & \left(x_{i} \mapsto \tilde{f}\left(x_{i}\right) x_{i}^{-1}\right)
\end{array}
$$

where $\tilde{f} \in \operatorname{End} F_{n}$ is a lift of $f$, defines a well-defined injective homomorphism by an argument similar to that in the proof of Lemma 5.6. Then we define a homomorphism $\widetilde{J}_{k}: \operatorname{Aut} F_{n}^{\text {acy }}[k] \rightarrow \operatorname{Hom}\left(F_{n},\left(\Gamma^{k} F_{n}\right) /\left(\Gamma^{2 k-1} F_{n}\right)\right)$ by the composite

$$
\operatorname{Aut} F_{n}^{\text {acy }}[k] \stackrel{\Phi_{2 k-1}}{\longrightarrow} \operatorname{Ker}\left(\operatorname{Aut} N_{2 k-1} \rightarrow \operatorname{Aut} N_{k}\right) \stackrel{\bar{J}_{k}}{\longrightarrow} \operatorname{Hom}\left(F_{n},\left(\Gamma^{k} F_{n}\right) /\left(\Gamma^{2 k-1} F_{n}\right)\right) .
$$

It is easily checked that $\widetilde{J}_{k}$ gives a refinement of the Johnson homomorphism and that the kernel of $\widetilde{J}_{k}$ coincides with Aut $F_{n}^{\text {acy }}[2 k-1]$. 
To show that $\widetilde{J}_{k}$ is surjective, we recall the direct sum decomposition $(* *)$. We write

$$
p_{l}:\left(\Gamma^{k} F_{n}\right) /\left(\Gamma^{2 k-1} F_{n}\right) \rightarrow\left(\Gamma^{k+l-1} F_{n}\right) /\left(\Gamma^{k+l} F_{n}\right), \quad(1 \leq l \leq k-1)
$$

for the $l^{\text {th }}$ projection. While each projection $p_{l}(2 \leq l \leq k-1)$ except $p_{1}$ is not given canonically, its restriction to $\left(\Gamma^{k+l-1} F_{n}\right) /\left(\Gamma^{2 k-1} F_{n}\right)$ coincides with the natural projection $\left(\Gamma^{k+l-1} F_{n}\right) /\left(\Gamma^{2 k-1} F_{n}\right) \rightarrow\left(\Gamma^{k+l-1} F_{n}\right) /\left(\Gamma^{k+l} F_{n}\right)$. Therefore, if we consider the isomorphism given by

$$
\operatorname{Hom}\left(F_{n},\left(\Gamma^{k} F_{n}\right) /\left(\Gamma^{2 k-1} F_{n}\right)\right) \underset{p_{1} \oplus \cdots \oplus p_{k-1}}{\cong} \bigoplus_{j=k}^{2 k-2} \operatorname{Hom}\left(F_{n},\left(\Gamma^{j} F_{n}\right) /\left(\Gamma^{j+1} F_{n}\right)\right),
$$

the composite

$$
\left.p_{l} \circ \widetilde{J}_{k}\right|_{\text {Aut } F_{n}^{\text {acy }}[k+l-1]}: \operatorname{Aut} F_{n}^{\text {acy }}[k+l-1] \rightarrow \operatorname{Hom}\left(F_{n},\left(\Gamma^{k+l-1} F_{n}\right) /\left(\Gamma^{k+l} F_{n}\right)\right)
$$

is nothing other than the original Johnson homomorphism $J_{k+l-1}$ for each $l=$ $1,2, \ldots, k-1$. Since $J_{k}, \ldots, J_{2 k-2}$ are all surjective, our claim follows.

Remark 5.7 The homomorphism $\widetilde{J}_{k}$ highly depends on the choice of a generating system of $F_{n}$, and $\widetilde{J}_{k}$ is not Aut $F_{n}^{\text {acy }}$-equivariant for $k \geq 3$. This phenomenon is explained by using the Magnus expansion as follows. It is well known that the expansion of an element of $\Gamma^{k} F_{n}$ has a form of $1+$ (degree $\geq k$-part). In terms of the Magnus expansion, our refinement $\widetilde{J}_{k}$ captures an information of the part from degree $k$ up to $(2 k-2)$ of the expansion of $\tilde{f}\left(x_{i}\right) x_{i}^{-1}$ under a fixed generating system of $F_{n}$. For a changing of a generating system, the Magnus expansion for each element intensively varies except that the first non-trivial homogeneous component in the positive degree part changes Aut $F_{n}^{\text {acy }}$-equivariantly (see [15], [9]).

The Magnus representation Here we define the Magnus representation for Aut $F_{n}^{\text {acy }}$. While we call it the Magnus "representation", it is actually a crossed homomorphism. The construction of the representation is based on Le Dimet's work [11], where the Gassner representation for the pure braid group is extended to that for the string link concordance group.

Before starting our discussion, we summarize our notation and rules. For a matrix $A$ with coefficients in a ring $R$, and a homomorphism $\varphi: R \rightarrow R^{\prime}$, we denote by ${ }^{\varphi} A$ the matrix obtained from $A$ by applying $\varphi$ to each entry. When $R=\mathbb{Z} G$ for a group $G$ (or its Cohn localization mentioned below), we denote by $\bar{A}$ the matrix obtained from $A$ by applying the involution induced from $\left(x \mapsto x^{-1}, x \in G\right)$ to each entry.

For a (finite) CW-complex $X$ and its regular covering $X_{\Gamma}$ with respect to a homomorphism $\pi_{1} X \rightarrow \Gamma, \Gamma$ acts on $X_{\Gamma}$ from the right through its deck transformation group. 
Therefore we regard the $\mathbb{Z} \Gamma$-cellular chain complex $C_{*}\left(X_{\Gamma}\right)$ of $X_{\Gamma}$ as a collection of free right $\mathbb{Z} \Gamma$-modules consisting of column vectors together with differentials given by left multiplications of matrices. For each $\mathbb{Z} \Gamma$-bimodule $A$, the twisted chain complex $C_{*}(X ; A)$ is given by the tensor product of the right $\mathbb{Z} \Gamma$-module $C_{*}\left(X_{\Gamma}\right)$ and the left $\mathbb{Z} \Gamma$-module $A$, so that $C_{*}(X ; A)$ and $H_{*}(X ; A)$ are right $\mathbb{Z} \Gamma$-modules.

To construct the Magnus representation for Aut $F_{n}^{\text {acy }}$, we use the following special case of the Cohn localization (or the universal localization). We refer to Section 7 in [2] for details.

Proposition 5.8 (Cohn [2]) Let $G$ be a group and let $\varepsilon: \mathbb{Z} G \rightarrow \mathbb{Z}$ be the augmentation map. Then there exists a pair of a ring $\Lambda_{G}$ and a ring homomorphism $l_{G}: \mathbb{Z} G \rightarrow \Lambda_{G}$ satisfying the following properties:

(1) For every matrix $m$ with coefficients in $\mathbb{Z} G$, if $\varepsilon(m)$ is invertible then $l_{G}(m)$ is also invertible.

(2) The pair $\left(\Lambda_{G}, l_{G}\right)$ is universal among all pairs having the property 1.

Furthermore it is unique up to isomorphism.

Example 5.9 When $G=H_{1}\left(F_{n}\right)$, we have

$$
\Lambda_{G} \cong\left\{\frac{f}{g} \mid f, g \in \mathbb{Z} G, \varepsilon(g)= \pm 1\right\} .
$$

We write $x_{i}$ again for the image of $x_{i}$ by $\iota_{F_{n}}: F_{n}=\left\langle x_{1}, x_{2}, \ldots, x_{n}\right\rangle \hookrightarrow F_{n}^{\text {acy }}$.

Proposition 5.10 (Proposition 1.1 in [11]) The homomorphism

$$
\begin{aligned}
v: \quad \Lambda_{F_{n}^{\text {acy }}}^{n} & \rightarrow I\left(F_{n}^{\text {acy }}\right) \otimes_{F_{n}^{\text {acy }}} \Lambda_{F_{n}^{\text {acy }}} \\
\Psi & \\
\left(a_{1}, \ldots, a_{n}\right) & \mapsto \sum_{i=1}^{n}\left(x_{i}^{-1}-1\right) \otimes a_{i}
\end{aligned}
$$

is an isomorphism of right $\Lambda_{F_{n}^{\text {acy }}}$-modules, where $I\left(F_{n}^{\text {acy }}\right):=\operatorname{Ker}\left(\varepsilon: \mathbb{Z} F_{n}^{\text {acy }} \rightarrow \mathbb{Z}\right)$.

Note that each automorphism of $F_{n}^{\text {acy }}$ induces one of $\mathbb{Z} F_{n}^{\text {acy }}$. Moreover, by the universal property of $\Lambda_{F_{n}^{\text {acy }}}$, an automorphism of $\Lambda_{F_{n}}^{\text {acy }}$ is also induced.

The proof of Proposition 5.10 is almost the same as that of Proposition 1.1 in [11], once we show the following. 
Lemma 5.11 Let $G$ be a finitely presentable group, and let $f: F_{n} \rightarrow G$ be a 2connected homomorphism. Then $f_{*}: H_{i}\left(F_{n} ; \Lambda_{G}\right) \rightarrow H_{i}\left(G ; \Lambda_{G}\right)$ is an isomorphism for $i=0,1,2$.

Proof We prove this lemma by using the idea of the proof of Proposition 2.1 in [10]. Let $X=K\left(F_{n}, 1\right)$ be a bouquet of $n$ circles and $Y=K(G, 1)$ be a CW-complex constructed from a finite presentation of $G$. The number of cells of $Y$ up to degree 2 is finite. We denote by $f$ again for the continuous map from $X$ to $Y$ induced by the homomorphism $f: F_{n} \rightarrow G$. Taking a mapping cylinder with respect to $f$, we obtain a CW-complex $M=K(G, 1)$ where $X$ is contained as a subcomplex. The number of cells of $M$ up to degree 2 is also finite. Since $H_{i}(M, X)=0$ for $i=0,1,2$, we can take a partial chain homotopy $D_{i+1}: C_{i}(M, X) \rightarrow C_{i+1}(M, X)$ of the partial chain complex $C_{3}(M, X) \rightarrow \cdots \rightarrow C_{0}(M, X) \rightarrow 0$ freely generated by relative cells of $(M, X)$. Namely, we have

$$
\begin{aligned}
& 1=\partial_{1} \circ D_{1}, \\
& 1=\partial_{2} \circ D_{2}+D_{1} \circ \partial_{1}, \\
& 1=\partial_{3} \circ D_{3}+D_{2} \circ \partial_{2} .
\end{aligned}
$$

Let $\tilde{M}$ be the universal covering of $M$ and $\tilde{X}$ be the inverse image of $X$ on $\tilde{M}$. We choose a lift of each cell of $M$ on $\tilde{M}$. Using the lifts of cells, we can define lifts $\widetilde{D}_{i+1}: C_{i}(\tilde{M}, \tilde{X}) \rightarrow C_{i+1}(\tilde{M}, \tilde{X})$ of the chain homotopy $D_{i+1}$ for $i=0,1,2$, which are $\mathbb{Z} G$-equivariant. Then we define

$$
\begin{aligned}
& \Phi_{0}:=\tilde{\partial_{1}} \circ \tilde{D_{1}}, \\
& \Phi_{1}:=\tilde{\partial_{2}} \circ \tilde{D_{2}}+\tilde{D_{1}} \circ \tilde{\partial_{1}}, \\
& \Phi_{2}:=\tilde{\partial}_{3} \circ \tilde{D_{3}}+\tilde{D_{2}} \circ \tilde{\partial_{2}},
\end{aligned}
$$

where $\partial_{i}$ are differentials of the chain complex $C_{i}(\tilde{M}, \tilde{X})$. It is easily checked that $\Phi_{i}: C_{i}(\tilde{M}, \tilde{X}) \rightarrow C_{i}(\tilde{M}, \tilde{X})(i=0,1,2)$ gives a partial chain map, so that it induces a homomorphism $\left(\Phi_{i}\right)_{*}: H_{i}(\tilde{M}, \tilde{X}) \rightarrow H_{i}(\tilde{M}, \tilde{X})$ for each $i=0,1,2$. Note that each $\Phi_{i}$ is a homomorphism between finitely generated free $\mathbb{Z} G$-modules which is the identity map on the base space. Then by the definition of the Cohn localization,

$$
\Phi_{i} \otimes_{G} 1: C_{i}(\tilde{M}, \tilde{X}) \otimes_{G} \Lambda_{G} \longrightarrow C_{i}(\tilde{M}, \tilde{X}) \otimes_{G} \Lambda_{G}
$$

becomes an isomorphism for each $i=0,1,2$. Moreover $\Phi_{i} \otimes_{G} 1$ maps $\operatorname{Ker}\left(\widetilde{\partial}_{i} \otimes_{G} 1\right)$ onto itself, so that $\left(\Phi_{i} \otimes_{G} 1\right)$ induces an epimorphism on $H_{i}$. 
On the other hand, since

$$
\begin{aligned}
& \Phi_{0} \otimes_{G} 1=\tilde{\partial_{1}} \circ \tilde{D_{1}} \otimes_{G} 1, \\
& \Phi_{1} \otimes_{G} 1=\left(\tilde{\partial_{2}} \circ \tilde{D_{2}}+\tilde{D_{1}} \circ \tilde{\partial_{1}}\right) \otimes_{G} 1, \\
& \Phi_{2} \otimes_{G} 1=\left(\tilde{\partial_{3}} \circ \tilde{D_{3}}+\tilde{D_{2}} \circ \tilde{\partial_{2}}\right) \otimes_{G} 1,
\end{aligned}
$$

we see that $\left(\Phi_{i} \otimes_{G} 1\right)_{*}: H_{i}\left(M, X ; \Lambda_{G}\right) \rightarrow H_{i}\left(M, X ; \Lambda_{G}\right)$ are 0 -maps, and therefore $H_{i}\left(M, X ; \Lambda_{G}\right)=0$ for $i=0,1,2$. Then $0=H_{2}\left(M ; \Lambda_{G}\right)=H_{2}\left(G ; \Lambda_{G}\right)$. From this, we see that $f_{*}: H_{i}\left(F_{n} ; \Lambda_{G}\right) \rightarrow H_{i}\left(G, \Lambda_{G}\right)$ is an isomorphism for each $i=0,1,2$.

Definition 5.12 For $1 \leq i \leq n$, we define a map $\partial / \partial x_{i}: F_{n}^{\text {acy }} \rightarrow \Lambda_{F_{n}^{\text {acy }}}$ by

$$
\begin{aligned}
\left(\frac{\partial}{\partial x_{1}}, \frac{\partial}{\partial x_{2}}, \ldots, \frac{\partial}{\partial x_{n}}\right): F_{n}^{\text {acy }} & \rightarrow \quad \Lambda_{F_{n}^{\text {acy }}}^{n} \\
\cup & \\
g & \mapsto \frac{\Psi}{v^{-1}\left(\left(g^{-1}-1\right) \otimes 1\right) .}
\end{aligned}
$$

The above maps $\partial / \partial x_{i}$ coincide with the ordinary free differentials if we restrict them to $F_{n}$, and have similar properties. We refer to Proposition 1.3 in [11]. In particular, we have

$$
\left(g^{-1}-1\right) \otimes 1=\sum_{i=1}^{n}\left(x_{i}^{-1}-1\right) \otimes \overline{\left(\frac{\partial g}{\partial x_{i}}\right)}
$$

for any $g \in F_{n}^{\text {acy }}$ under our notation.

Definition 5.13 We define the Magnus representation

$$
\begin{aligned}
& r: \text { Aut } F_{n}^{\text {acy }} \rightarrow M\left(n, \Lambda_{F_{n}^{\text {acy }}}\right) \\
& r(\varphi):=\left(\overline{\left(\frac{\partial \varphi\left(x_{j}\right)}{\partial x_{i}}\right)}\right)_{i, j}
\end{aligned}
$$

by setting

for $\varphi \in \operatorname{Aut} F_{n}^{\text {acy }}$

Proposition 5.14 The Magnus representation $r$ is a crossed homomorphism. In particular, the image of $r$ is contained in the set of invertible matrices.

Proof For $\varphi, \psi \in \operatorname{Aut} F_{n}^{\text {acy }}$, we have

$$
\left(\varphi \psi\left(x_{j}^{-1}\right)-1\right) \otimes 1=\sum_{i=1}^{n}\left(x_{i}^{-1}-1\right) \otimes \overline{\left(\frac{\partial \varphi \psi\left(x_{j}\right)}{\partial x_{i}}\right)}
$$


by definition. On the other hand,

$$
\begin{aligned}
\left(\varphi \psi\left(x_{j}^{-1}\right)-1\right) \otimes 1 & ={ }^{\varphi}\left(\left(\psi\left(x_{j}^{-1}\right)-1\right) \otimes 1\right) \\
& =\sum_{k=1}^{\varphi}\left(x_{k}^{-1}-1\right) \otimes \overline{\left.\left(\frac{\partial \psi\left(x_{j}\right)}{\partial x_{k}}\right)\right)} \\
& =\sum_{k=1}^{n}\left(\varphi\left(x_{k}^{-1}\right)-1\right) \otimes \overline{\left(\frac{\partial \psi\left(x_{j}\right)}{\partial x_{k}}\right)} \\
& =\sum_{k=1}^{n}\left\{\left(\varphi\left(x_{k}^{-1}\right)-1\right) \otimes 1\right\} \cdot \overline{\left(\frac{\partial \psi\left(x_{j}\right)}{\partial x_{k}}\right)} \\
& =\sum_{k=1}^{n}\left\{\sum_{i=1}^{n}\left(x_{i}^{-1}-1\right) \otimes \overline{\left(\frac{\partial \varphi\left(x_{k}\right)}{\partial x_{i}}\right)}\right\} \cdot \frac{\left(\frac{\partial \psi\left(x_{j}\right)}{\partial x_{k}}\right)}{\varphi} \cdot \frac{\left(\frac{\partial \psi\left(x_{j}\right)}{\partial x_{k}}\right)}{\left(\frac{\left.\partial x_{k}\right)}{\partial x_{i}}\right)} \cdot \\
& =\sum_{i=1}^{n}\left(x_{i}^{-1}-1\right) \otimes\left\{\sum_{k=1}^{n} \cdot\right.
\end{aligned}
$$

Hence we obtain

$$
\overline{\frac{\partial \varphi \psi\left(x_{j}\right)}{\partial x_{i}}}=\sum_{k=1}^{n} \overline{\left(\frac{\partial \varphi\left(x_{k}\right)}{\partial x_{i}}\right)} \cdot \varphi \overline{\left(\frac{\partial \psi\left(x_{j}\right)}{\partial x_{k}}\right)}
$$

which shows that $r(\varphi \psi)=r(\varphi) \cdot{ }^{\varphi} r(\psi)$.

Note that the composite $\mathbb{Z} F_{n} \stackrel{\iota_{F n}}{\longrightarrow} \mathbb{Z} F_{n}^{\text {acy }} \stackrel{l_{F_{n}}^{\text {acy }}}{\longrightarrow} \Lambda_{F_{n}^{\text {acy }}}$ is injective, for the composite of the ring homomorphism $\mathbb{Z} F_{n}^{\text {acy }} \rightarrow \mathbb{Z} F_{n}^{\text {nil }}$ with the Magnus expansion, which can be extended to $\mathbb{Z} F_{n}^{\text {nil }}$ and is injective on $\mathbb{Z} F_{n}$, satisfies the property 1 of Proposition 5.8,

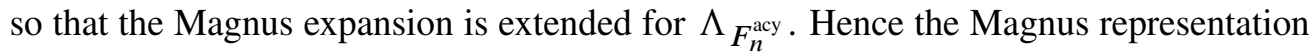
defined here certainly gives a generalization of the original $r$ : Aut $F_{n} \rightarrow G L\left(n, \mathbb{Z} F_{n}\right)$.

Example 5.15 Consider the 2-connected endomorphism $\psi$ in Example 3.2. Then

$$
r(\psi)=\left(\begin{array}{cc}
1+x_{2}^{-1} x_{1}^{-1}-x_{1} x_{2} x_{1}^{-1} x_{2}^{-1} x_{1}^{-1} & 0 \\
x_{1}^{-1}-x_{2} x_{1}^{-1} x_{2}^{-1} x_{1}^{-1} & 1
\end{array}\right) .
$$

Reducing the coefficients to $\Lambda_{H_{1}\left(F_{2}^{\text {acy }}\right)}=\Lambda_{H_{1}\left(F_{2}\right)}$, we obtain the matrix

$$
\left(\begin{array}{cc}
1+x_{1}^{-1} x_{2}^{-1}-x_{1}^{-1} & 0 \\
x_{1}^{-1}-x_{1}^{-2} & 1
\end{array}\right)
$$

Algebraic $8 \mathcal{G}$ Geometric Topology, Volume 6 (2006) 
whose determinant is $1+x_{1}^{-1} x_{2}^{-1}-x_{1}^{-1}$. Since this value is not invertible in $\mathbb{Z} H_{1}\left(F_{2}\right)$, we see that $\psi \notin$ Aut $F_{2}$.

\section{Main results}

In this section, we return to our discussion on homology cylinders, and consider some relationships between homology cylinders and the acyclic closure of a free group.

For each homology cylinder $\left(M, i_{+}, i_{-}\right) \in \mathcal{C}_{g, 1}$, we obtain a commutative diagram

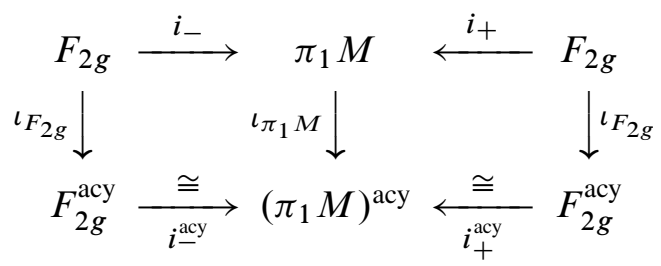

by Proposition 4.7. From this, we obtain a monoid homomorphism defined by

$$
\sigma^{\text {acy }}: \mathcal{C}_{g, 1} \longrightarrow \operatorname{Aut} F_{2 g}^{\text {acy }} \quad\left(\left(M, i_{+}, i_{-}\right) \mapsto\left(i_{+}^{\text {acy }}\right)^{-1} \circ i_{-}^{\text {acy }}\right)
$$

and it induces a group homomorphism $\sigma^{\text {acy }}: \mathcal{H}_{g, 1} \rightarrow$ Aut $F_{2 g}^{\text {acy }}$.

Our first result is a generalization of Dehn-Nielsen's theorem. Recall that $\zeta \in F_{2 g} \subset$ $F_{2 g}^{\text {acy }}$ is a word corresponding to the boundary loop of $\Sigma_{g, 1}$.

Theorem 6.1 The image of $\sigma^{\text {acy }}: \mathcal{H}_{g, 1} \rightarrow \operatorname{Aut} F_{2 g}^{\text {acy }}$ is

$$
\operatorname{Aut}_{0} F_{2 g}^{\text {acy }}:=\left\{\varphi \in \operatorname{Aut} F_{2 g}^{\text {acy }} \mid \varphi(\zeta)=\zeta \in F_{2 g}^{\text {acy }}\right\} .
$$

Proof By the definition of homology cylinders, we have $i_{+}(\zeta)=i_{-}(\zeta) \in \pi_{1} M$ for every homology cylinder $\left(M, i_{+}, i_{-}\right)$. Hence we see that the image of $\sigma^{\text {acy }}$ is contained in $\mathrm{Aut}_{0} F_{2 g}^{\mathrm{acy}}$.

Conversely, given an element $\varphi \in$ Aut $_{0} F_{2 g}^{\text {acy }}$, we construct a homology cylinder $M=$ $\left(M, i_{+}, i_{-}\right)$satisfying $\sigma^{\text {acy }}(M)=\varphi$. The construction is based on that of Theorem 2.3 (which is Theorem 3 in [3]). In our context, however, we must pay an extra attention because we do not have a result which directly corresponds to Lemma 4.6 in [3]. This occurs from the fact that $\iota_{F_{2 g}}: F_{2 g} \rightarrow F_{2 g}^{\text {acy }}$ is injective although the composite $F_{2 g} \rightarrow F_{2 g}^{\text {acy }} \rightarrow F_{2 g}^{\text {acy }} /\left(\Gamma^{k} F_{2 g}^{\text {acy }}\right) \cong N_{k}$ is surjective.

We begin by taking two continuous maps $f_{+}, f_{-}: \Sigma_{g, 1} \rightarrow K\left(F_{2 g}^{\text {acy }}, 1\right)$ corresponding to homomorphisms $\iota_{F_{2 g}}, \varphi \circ \iota_{F_{2 g}}: F_{2 g} \rightarrow F_{2 g}^{\text {acy }}$, respectively. Since $\iota_{F_{2 g}}(\zeta)=\varphi \circ \iota_{F_{2 g}}(\zeta)$, 
we can combine the two maps and obtain a map $f:=f_{+} \cup f_{-}: \Sigma_{2 g}=\Sigma_{g, 1} \cup$ $\left(-\Sigma_{g, 1}\right) \rightarrow K\left(F_{2 g}^{\text {acy }}, 1\right)$. The pair $\left(\Sigma_{2 g}, f\right)$ defines an element of the second bordism group $\Omega_{2}\left(F_{2 g}^{\text {acy }}\right)$ of $K\left(F_{2 g}^{\text {acy }}, 1\right)$, which is naturally isomorphic to $H_{2}\left(F_{2 g}^{\text {acy }}\right)$. Since $H_{2}\left(F_{2 g}^{\text {acy }}\right)=0$ as mentioned in Proposition 4.6, there exist a compact oriented 3manifold $M$ whose boundary is $\Sigma_{2 g}$, and a map $\Phi: M \rightarrow K\left(F_{2 g}^{\text {acy }}, 1\right)$ extending the map $f$. We write $i_{+}, i_{-}: \Sigma_{g, 1} \rightarrow \partial M$ for embeddings onto domains of $f_{+}, f_{-}$, respectively.

Since $\iota_{F_{2 g}}$ is 2-connected, we have $H_{1}(M) \cong i_{+}\left(H_{1}\left(\Sigma_{g, 1}\right)\right) \oplus \operatorname{Ker} \Phi_{*}$. If $\operatorname{Ker} \Phi_{*}=0$, then $i_{+}, i_{-}: H_{1}\left(\Sigma_{g, 1}\right) \rightarrow H_{1}(M)$ are both isomorphisms. In particular, $H_{1}(M, \partial M)=$ 0 and therefore $H^{1}(M, \partial M) \cong H_{2}(M)=0$, so that the triplet $\left(M, i_{+}, i_{-}\right)$gives a homology cylinder satisfying $\sigma^{\text {acy }}(M)=\varphi$. If not, we perform surgery on the map $\Phi$ to kill $\operatorname{Ker} \Phi_{*}$.

Now we take a non-trivial element $\alpha \in \operatorname{Ker} \Phi_{*}$. If there exists a representative $C \in \pi_{1} M$ by a simple closed curve such that $\Phi(C)=1 \in F_{2 g}^{\text {acy }}$, then we can do surgery on $C$ and extend $\Phi$ over the trace of the surgery. However, we cannot necessarily take such a simple closed curve. Then we replace $\left(M, i_{+}, i_{-}\right)$by a manifold which is homology bordant to $M$ over $K\left(F_{2 g}^{\text {acy }}, 1\right)$ and for which we can take a simple closed curve which represents $\alpha$ and whose image by $\Phi$ is trivial in $F_{2 g}^{\text {acy }}$. A construction of such a homology bordant manifold is given as follows.

For the induced homomorphism $i_{+}: F_{2 g} \rightarrow \pi_{1} M$, by the universal property of acyclic closures, we have a homomorphism $i_{+}^{\text {acy }}: F_{2 g}^{\text {acy }} \rightarrow\left(\pi_{1} M\right)^{\text {acy }}$ satisfying $i_{+}^{\text {acy }} \circ \iota_{F_{2 g}}=$ $\iota_{\pi_{1} M} \circ i_{+}$. Similarly we have $\Phi^{\text {acy }}:\left(\pi_{1} M\right)^{\text {acy }} \rightarrow F_{2 g}^{\text {acy }}$ satisfying $\Phi=\Phi^{\text {acy }} \circ \iota_{\pi_{1}} M$. Then $\Phi^{\text {acy }} \circ i_{+}^{\text {acy }} \circ \iota_{F_{2 g}}=\Phi^{\text {acy }} \circ \iota_{\pi_{1}} M \circ i_{+}=\iota_{F_{2 g}}$, so that $\Phi^{\text {acy }} \circ i_{+}^{\text {acy }}=\mathrm{id}_{F_{2 g}^{\text {acy }}}$. In particular, $\Phi^{\text {acy }}$ is onto.

Take a simple closed curve $C$ representing $\alpha \in \operatorname{Ker} \Phi_{*}$. Since $\Phi_{*}(\alpha)=0 \in H_{1}\left(F_{2 g}^{\text {acy }}\right)$, we can write $\Phi(C)=\prod_{i=1}^{l}\left[h_{i 1}, h_{i 2}\right]$ with $h_{i j} \in F_{2 g}^{\text {acy }}$. We take an acyclic system

$$
S: x_{i}=w_{i}\left(x_{1}, x_{2}, \ldots, x_{m}\right) \quad(i=1,2, \ldots, m)
$$

over $\pi_{1} M$ whose solution in $\left(\pi_{1} M\right)^{\text {acy }}$ contains

$$
\left\{i_{+}^{\text {acy }}\left(h_{11}\right), i_{+}^{\text {acy }}\left(h_{12}\right), \ldots, i_{+}^{\text {acy }}\left(h_{l 1}\right), i_{+}^{\text {acy }}\left(h_{l 2}\right)\right\} .
$$

We attach a 1-handle to $M \times\{1\} \subset M \times I$ for each variable $x_{i}$ and write $x_{i}$ again for the added generator on the fundamental group of the resulting cobordism. We also attach a 2 -handle along the loop $x_{i} w_{i}^{-1}$ for each $i=1,2, \ldots, m$ with any framing. We denote the resulting cobordism by $X_{S}$. Then

$$
\pi_{1} X_{S}=\left(\pi_{1} M *\left\langle x_{1}, \ldots, x_{m}\right\rangle\right) /\left(x_{1} w_{1}^{-1}, \ldots, x_{m} w_{m}^{-1}\right) .
$$


Let $M_{S}$ be another part of the boundary of $X_{S}$, namely $M \cup M_{S}=\partial X_{S}$ and $M \cap M_{S}=\partial M=\partial M_{S}$. We define a homomorphism $\Phi_{S}: \pi_{1} X_{S} \rightarrow\left(\pi_{1} M\right)^{\text {acy }}$ which lifts $\iota_{\pi_{1} M}$ by sending $x_{i}$ to the corresponding solution of $S$. Then the composite $\Phi^{\text {acy }} \circ \Phi_{S}: \pi_{1} X_{S} \rightarrow F_{2 g}^{\text {acy }}$ gives a continuous map $\Phi_{S}: X_{S} \rightarrow K\left(F_{2 g}^{\text {acy }}, 1\right)$ which extends $\Phi: M \rightarrow K\left(F_{2 g}^{\text {acy }}, 1\right)$.

Since $\iota_{\pi_{1} M}$ is 2-connected and has a lift $\Phi_{S}$, the homomorphism $H_{1}(M) \rightarrow H_{1}\left(X_{S}\right)$ induced from the inclusion $M \hookrightarrow X_{S}$ is injective. On the other hand, by the definition of $X_{S}$, this homomorphism is onto, hence an isomorphism. We can also see that $H_{2}(M) \rightarrow H_{2}\left(X_{G}\right)$ is onto, for, in terms of chain complexes consisting of handles, the boundary of each newly added 2-handle corresponding to the relation $x_{i} w_{i}^{-1}$, has a form of $\left[x_{i}\right]+(1-$ handles in $M \times I)$ which shows that we have a surjective homomorphism from the module of 2-cycles on $M \times I$ to that on $X_{S}$.

We also show that $H_{1}\left(M_{S}\right) \rightarrow H_{1}\left(X_{S}\right)$ is an isomorphism. The surjectivity follows by considering the dual handle decomposition of $X_{S}$, namely $X_{S}$ is constructed from $M_{S} \times I$ by attaching 3- and 2-handles. To show the injectivity, we see that $H_{2}\left(X_{S}, M_{S}\right)=0$. By the Poincaré-Lefschetz duality, $H_{2}\left(X_{S}, M_{S}\right) \cong H^{2}\left(X_{S}, M\right)$. On the other hand, we have an exact sequence

$$
H^{1}\left(X_{S}\right) \longrightarrow H^{1}(M) \longrightarrow H^{2}\left(X_{S}, M\right) \longrightarrow H^{2}\left(X_{S}\right) \longrightarrow H^{2}(M) \text {. }
$$

Since $H_{*}(M) \rightarrow H_{*}\left(X_{S}\right)$ is an isomorphism for $*=1$ and onto for $*=2$, the first map is an isomorphism, and the last one is injective. Hence $H^{2}\left(X_{S}, M\right)=0$.

From the above argument, we see that $M$ and $M_{S}$ are homology bordant over $K\left(F_{2 g}^{\text {acy }}, 1\right)$ by the bordism $X_{S}$ and $\Phi_{S}$. Note that this bordism preserves the direct sum decomposition $H_{1}(M) \cong i_{+}\left(H_{1}\left(\Sigma_{g, 1}\right)\right) \oplus \operatorname{Ker} \Phi_{*}$, namely we also have $H_{1}\left(M_{S}\right) \cong i_{+}\left(H_{1}\left(\Sigma_{g, 1}\right)\right) \oplus \operatorname{Ker} \Phi_{G *}$ and we can take $\bar{\alpha} \in \operatorname{Ker} \Phi_{G *}$ which corresponds to $\alpha$. Consider the simple closed curve $C$ taken at the beginning of this argument. Since $\pi_{1} M_{S} \rightarrow \pi_{1} X_{S}$ is onto, there exists a simple closed curve $\bar{C}$ which attains $C$ in $\pi_{1} X_{S}$. Now $h_{i j} \in F_{2 g}^{\text {acy }}$ are contained in the image of $\Phi_{S}: \pi_{1} M_{S} \rightarrow F_{2 g}^{\text {acy }}$, so that we can take $\overline{h_{i j}} \in \pi_{1} M_{S}$ attaining $h_{i j}$. Then the simple closed curve $\bar{C}\left(\prod_{i=1}^{l}\left[\overline{h_{i 1}}, \overline{h_{i 2}}\right]\right)^{-1}$ represents $\bar{\alpha}$ and is mapped by $\Phi_{S}$ to the trivial element of $F_{2 g}^{\text {acy }}$.

The rest of the proof is almost the same as Theorem 3 in [3]. The difference is that our killing $\operatorname{Ker} \Phi_{*}$ goes, if necessary, with changing the manifold to some homology bordant one at each step.

Next, we consider $\mathcal{H}_{g, 1}[[\infty]]:=\operatorname{Ker}\left(\sigma^{\text {acy }}: \mathcal{H}_{g, 1} \rightarrow\right.$ Aut $\left._{0} F_{2 g}^{\text {acy }}\right)$. In contrast to the case of the mapping class group, $\mathcal{H}_{g, 1}[[\infty]]$ is non-trivial. Indeed the homology cobordism 
group $\Theta_{\mathbb{Z}}^{3}$ of homology $3-$ spheres is contained in it. Our second result gives an additive invariant for $\mathcal{H}_{g, 1}[[\infty]]$.

For any element $\left[\left(M, i_{+}, i_{-}\right)\right] \in \mathcal{H}_{g, 1}[[\infty]]$, we have $i_{+}^{\text {acy }}=i_{-}^{\text {acy }}: F_{2 g}^{\text {acy }} \rightarrow\left(\pi_{1} M\right)^{\text {acy }}$. Consider the composite $f: M \rightarrow K\left(F_{2 g}^{\text {acy }}, 1\right)$ of continuous maps

$$
M \longrightarrow K\left(\pi_{1} M, 1\right) \longrightarrow K\left(\left(\pi_{1} M\right)^{\text {acy }}, 1\right) \longrightarrow K\left(F_{2 g}^{\text {acy }}, 1\right)
$$

where the last map is induced by the homomorphism $\left(i_{+}^{\text {acy }}\right)^{-1}=\left(i_{-}^{\text {acy }}\right)^{-1}$. After adjusting by some homotopy, if necessary, we have $f \circ i_{+}=f \circ i_{-}: \Sigma_{g, 1} \rightarrow K\left(F_{2 g}^{\text {acy }}, 1\right)$. Then we can consider a closed 3-manifold

$$
C_{M}=M /\left(i_{+}(x)=i_{-}(x)\right) \quad x \in \Sigma_{g, 1}
$$

and a continuous map $\widehat{f}: C_{M} \rightarrow K\left(F_{2 g}^{\text {acy }}, 1\right)$ induced from $f$. Note that $C_{M}$ is also obtained by gluing $1_{\mathcal{C}_{g, 1}}=\Sigma_{g, 1} \times I$ along their boundaries. We call this operation closing.

Theorem 6.2 The map $\theta: \mathcal{H}_{g, 1}[[\infty]] \rightarrow H_{3}\left(F_{2 g}^{\text {acy }}\right)$ given by

$$
\theta\left(\left[M, i_{+}, i_{-}\right]\right):=\widehat{f}\left(\left[C_{M}\right]\right) \quad \in H_{3}\left(F_{2 g}^{\text {acy }}\right)
$$

is a well-defined homomorphism. Moreover it is surjective.

Proof The proof is divided into three steps:

- $\widehat{f}\left(\left[C_{M}\right]\right)$ depends only on the homology cobordism class of $\left(M, i_{+}, i_{-}\right)$,

- $\theta$ is actually a homomorphism,

- $\theta$ is onto.

Step 1 Let $\left(M, i_{+}, i_{-}\right)$and $\left(N, j_{+}, j_{-}\right)$be homology cylinders contained in the same homology cobordism class in $\mathcal{H}_{g, 1}[[\infty]]$. We write $f_{M}: M \rightarrow K\left(F_{2 g}^{\text {acy }}, 1\right)$ and $f_{N}: N \rightarrow K\left(F_{2 g}^{\text {acy }}, 1\right)$ for maps constructed as above, respectively. We take a homology cobordism $W$ satisfying

$$
\partial W=M \cup(-N) /\left(i_{+}(x)=j_{+}(x), i_{-}(x)=j_{-}(x)\right) \quad x \in \Sigma_{g, 1},
$$

so that we have $i_{+}=j_{+}, i_{-}=j_{-}: \Sigma_{g, 1} \rightarrow W$ and

$$
i_{+}^{\text {acy }}=j_{+}^{\text {acy }}=i_{-}^{\text {acy }}=j_{-}^{\text {acy }}: F_{2 g}^{\text {acy }} \rightarrow\left(\pi_{1} W\right)^{\text {acy }} .
$$

Note that, by Stallings' theorem,

$$
F_{2 g}^{\text {acy }} \cong\left(\pi_{1} M\right)^{\text {acy }} \cong\left(\pi_{1} W\right)^{\text {acy }} \cong\left(\pi_{1} N\right)^{\text {acy }} .
$$

Algebraic $8 \mathcal{G}$ Geometric Topology, Volume 6 (2006) 
Then the homomorphism $\left(i_{+}^{\text {acy }}\right)^{-1} \circ \iota_{\pi_{1} W}\left(=\left(j_{+}^{\text {acy }}\right)^{-1} \circ \iota_{\pi_{1} W}\right)$ induces a map $f_{W}: W \rightarrow$ $K\left(F_{2 g}^{\text {acy }}, 1\right)$ extending $f_{M}$ and $f_{N}$.

We take a closed tubular neighborhood $V$ of $\partial M=\partial N$ in $\partial W . V$ is diffeomorphic to $\Sigma_{2 g} \times I$. We glue $1_{\mathcal{C}_{g, 1}} \times I=\left(\Sigma_{g, 1} \times I\right) \times I$ to $W$ by identifying $\left(\partial 1_{\mathcal{C}_{g, 1}}\right) \times I$ with $V$. The resulting 4-manifold $\widehat{W}$ gives a homology cobordism between $C_{M}$ and $C_{N}$. Moreover, for $f_{M} \circ i_{+}=f_{N} \circ j_{+}$and $f_{M} \circ i_{-}=f_{N} \circ j_{-}$are homotopic, the map $f_{W}$ is extended to a map $\widehat{f}_{W}: \widehat{W} \rightarrow K\left(F_{2 g}^{\text {acy }}, 1\right)$ whose restriction to $C_{M}$ (resp. $C_{N}$ ) coincides with $\widehat{f}_{M}$ (resp. $\left.\widehat{f}_{N}\right)$. Therefore we see $\widehat{f}_{M}\left(\left[C_{M}\right]\right)=\widehat{f}_{N}\left(\left[C_{N}\right]\right)$.

Step 2 For $\left[\left(M, i_{+}, i_{-}\right)\right],\left[\left(N, j_{+}, j_{-}\right)\right] \in \mathcal{H}_{g, 1}[[\infty]]$, we construct a 4-manifold

$$
W=(M \times I) \cup(N \times I) \cup\left(1_{\mathcal{C}_{g, 1}} \times[0,3]\right)
$$

by the following gluing rule. We decompose $\partial\left(1_{\mathcal{C}_{g, 1}} \times[0,3]\right)$ into

$$
\left(\left(\partial 1_{\mathcal{C}_{g, 1}}\right) \times[0,1]\right) \cup\left(\left(\partial 1_{\mathcal{C}_{g, 1}}\right) \times[1,2]\right) \cup\left(\left(\partial 1_{\mathcal{C}_{g, 1}}\right) \times[2,3]\right) \cup\left(1_{\mathcal{C}_{g, 1}} \times\{0,3\}\right) .
$$

We glue $\left(\partial 1_{\mathcal{C}_{g, 1}}\right) \times[0,1]$ to $(\partial M) \times I$. We also glue $\left(\partial 1_{\mathcal{C}_{g, 1}}\right) \times[2,3]$ to $\partial N \times I$ with opposite direction of unit intervals and opposite markings of homology cylinders $N$ and $1_{\mathcal{C}_{g, 1}}$. Namely, for example, we identify $\left(\Sigma_{g, 1} \times\{1\}\right) \times\{0\}$ with $i_{+}\left(\Sigma_{g, 1}\right) \times\{0\} \subset M \times I$, and $\left(\Sigma_{g, 1} \times\{0\}\right) \times\{3\}$ with $j_{+}\left(\Sigma_{g, 1}\right) \times\{0\} \subset N \times I$. Then $\partial W$ consists of $C_{M}, C_{N}$, and

$$
(-M \times\{1\}) \cup(-N \times\{1\}) \cup\left(\left(\partial 1_{\mathcal{C}_{g, 1}}\right) \times[1,2]\right) .
$$

The last component is nothing other than $-C_{M \cdot N}$. Hence $W$ is a cobordism between $C_{M} \sqcup C_{N}$ and $-C_{M \cdot N}$.

Since $f_{M} \circ i_{+}, f_{M} \circ i_{-}, f_{N} \circ j_{+}$and $f_{N} \circ j_{-}$are all homotopic, $\left(f_{M} \times I\right) \cup\left(f_{N} \times\right.$ $I):(M \times I) \cup(N \times I) \rightarrow K\left(F_{2 g}^{\text {acy }}, 1\right)$ is extended to a map $f_{W}: W \rightarrow K\left(F_{2 g}^{\text {acy }}, 1\right)$ whose restriction to $C_{M}, C_{N}$ and $-C_{M \cdot N}$ coincide with $\widehat{f}_{M}, \widehat{f}_{N}$ and $\widehat{f}_{M \cdot N}$, respectively. Hence we see that $\theta(M)+\theta(N)=\theta(M \cdot N)$.

Step 3 We construct a homology cylinder which attains a given element $\alpha \in H_{3}\left(F_{2 g}^{\text {acy }}\right)$ by $\theta$. First, we use a construction used in [16], [12].

Take a bouquet of $2 g$ circles tied at the base point $*$ as a model of $K\left(F_{2 g}, 1\right)$, and take a CW-complex realizing $K\left(F_{2 g}^{\text {acy }}, 1\right)$. The homomorphism $\iota_{F_{2 g}}: F_{2 g}=\left\langle\gamma_{1}, \ldots, \gamma_{2 g}\right\rangle \rightarrow$ $F_{2 g}^{\text {acy }}$ induces a continuous map $I_{F_{2 g}}: K\left(F_{2 g}, 1\right) \rightarrow K\left(F_{2 g}^{\text {acy }}, 1\right)$. As usual, we can assume that $K\left(F_{2 g}, 1\right)$ is a subcomplex of $K\left(F_{2 g}^{\text {acy }}, 1\right)$ having only one 0 -cell $*$, namely $I_{F_{2 g}}$ is an inclusion. Let $\widehat{K}$ be the mapping cone of $I_{F_{2 g}}$, which is obtained 
from the CW-complex $K\left(F_{2 g}^{\text {acy }}, 1\right)$ by attaching a 2-cell $e_{i}$ along each loop representing $\gamma_{i} \in F_{2 g} \subset F_{2 g}^{\text {acy }}$ for $i=1, \ldots, 2 g$. We take an interior point $p_{i}$ in each 2-cell $e_{i}$.

Since $H_{3}\left(F_{2 g}^{\text {acy }}\right)=H_{3}\left(K\left(F_{2 g}^{\text {acy }}, 1\right)\right) \cong H_{3}(\widehat{K}) \cong \Omega_{3}(\widehat{K})$, we can take a pair of a closed oriented 3-manifold $M$ and a continuous map $f: M \rightarrow \widehat{K}$ corresponding to $\alpha$ by this isomorphism. By applying a method used in the proof of Theorem 4 in [16] or Theorem 2 in [12], we can construct a pair of a closed oriented 3-manifold $M^{\prime}$ and a continuous map $f^{\prime}: M^{\prime} \rightarrow \widehat{K}$ satisfying the following.

- $\left(M^{\prime}, f^{\prime}\right)$ is bordant to $(M, f)$ over $\widehat{K}$, that is, $f^{\prime}\left(\left[M^{\prime}\right]\right)=f([M]) \in H_{3}(\widehat{K})$.

- $p_{i}$ is a regular value of $f^{\prime}$ for each $i=1, \ldots, 2 g$.

- $L_{i}:=\left(f^{\prime}\right)^{-1}\left(p_{i}\right)$ is not empty and is a knot in $M^{\prime}$ for each $i=1, \ldots, 2 g$.

- There exists a closed tubular neighborhood $T_{i}$ of $L_{i}$ such that the diagram

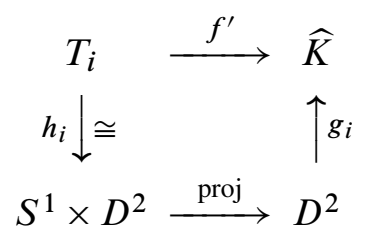

commutes where $h_{i}$ is a homeomorphism sending $L_{i} \subset T_{i}$ onto $S^{1} \times\{0\}$, and $g_{i}$ is a homeomorphism onto the 2 -cell $e_{i}$ sending $1 \in D^{2}$ to the base point $*$ of $\widehat{K}$.

For simplicity, we write $(M, f)$ again for $\left(M^{\prime}, f^{\prime}\right)$. We take a meridian loop $m_{i}:=$ $h_{i}^{-1}\left(\{1\} \times \partial D^{2}\right)$ and a point $q_{i}:=h_{i}^{-1}(1,1)$ on $m_{i}$ for each $i$. Note that $f\left(q_{i}\right)=*$. We orient $m_{i}$ by using the orientation of the loop representing $\gamma_{i}$.

We take a base point $q \in M \backslash\left(\bigcup_{i=1}^{2 g} T_{i}\right) \subset M$. By using some homotopy, we can assume $f(q)=*$. We connect the points $q_{i}$ and $q_{g+i}$ by a path $l_{i}^{1}$ in $M \backslash\left(\bigcup_{i=1}^{2 g} T_{i}\right)$ such that $f$ is the constant map to $*$ on a neighborhood of $l_{i}^{1}$. We also connect a point of $l_{i}^{1}$ and $q$ by a path $l_{i}^{2}$ satisfying the same condition for $f$ as $l_{i}^{1}$. The condition for $f$ on the neighborhood of $l_{i}^{1}, l_{i}^{2}$ will be satisfied by surgeries using 1 -handles. The meridian loops $m_{i}$ and the paths $l_{i}^{1}, l_{i}^{2}$ form a graph with trivalent vertices except one $g$-valent vertex $q$ as depicted below. 


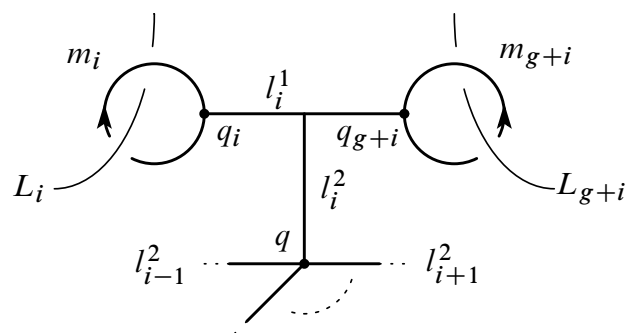

In a tubular neighborhood of this graph, we take an embedded ribbon whose boundary is connected and is sent by $f$ to the loop $\zeta=\prod_{i=1}^{g}\left[\gamma_{i}, \gamma_{g+i}\right]$ in $\widehat{K}$ as follows.

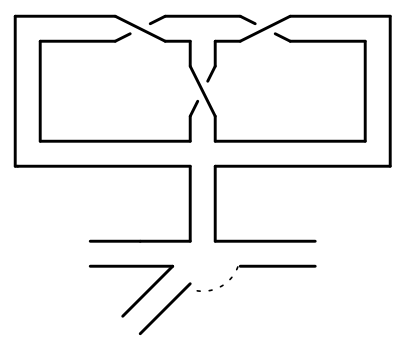

We fatten the ribbon to construct an embedding of $1_{\mathcal{C}_{g, 1}}=\Sigma_{g, 1} \times I$, in which the boundary of the ribbon divides $\partial 1_{\mathcal{C}_{g, 1}} \cong \Sigma_{g, 1} \cup\left(-\Sigma_{g, 1}\right)$ into two embeddings $i_{+}, i_{-}$ of $\Sigma_{g, 1}$.

We perform surgery at $L_{1}, L_{2}, \ldots, L_{2 g}$ with framings given by $\left(h_{i}\right)^{-1}\left(S^{1} \times\{1\}\right)$, on which $f$ is the constant map to $*$, and denote the resulting manifold by $M_{L}$. By the choice of framings, there exists a map $f_{L}: M_{L} \rightarrow K\left(F_{2 g}^{\text {acy }}, 1\right) \subset \widehat{K}$ which is bordant to $(M, f)$ over $\widehat{K}$. Note that $f_{L}\left(\left[M_{L}\right]\right)=\alpha \in H_{3}\left(K\left(F_{2 g}^{\text {acy }}, 1\right)\right)$.

We remove the embedded $1_{\mathcal{C}_{g, 1}}$ from $M_{L}$ to construct a 3-manifold $N$ with boundary. $\partial N$ is diffeomorphic to $\Sigma_{g, 1} \cup\left(-\Sigma_{g, 1}\right)$, and has an orientation preserving embedding $i_{+}$and a reversing one $i_{-}$. Now $f_{L} \circ i_{+}=f_{L} \circ i_{-}=\iota_{F_{2 g}}: \pi_{1} \Sigma_{g, 1} \cong F_{2 g} \rightarrow F_{2 g}^{\text {acy }}$, so that $f_{L} \circ i_{+}$and $f_{L} \circ i_{-}$are 2 -connected homomorphisms preserving $\zeta$. Only we have to do is to construct a homology cylinder from the data $N, i_{+}, i_{-}: \Sigma_{g, 1} \rightarrow \partial N$ and $f_{L}: N \rightarrow K\left(F_{2 g}^{\text {acy }}, 1\right)$ with keeping its bordism class over $K\left(F_{2 g}^{\text {acy }}, 1\right)$, which is the same situation of the proof of Theorem 6.1. Using the same argument, we obtain a desired homology cylinder.

Remark 6.3 As seen in Step 1 of the above proof, two homology cylinders belonging to the same class in $\mathcal{H}_{g, 1}$ give homology cobordant closed 3-manifolds by closing. 
Remark 6.4 We do not know, at present, whether $H_{3}\left(F_{2 g}^{\text {acy }}\right)$ is trivial or not. This situation is similar to that of some (string) link concordance invariant using the algebraic closure of a free group in [12] and [13]. It is easily checked that the homomorphism $\theta$ is trivial if we restrict it to $\Theta_{\mathbb{Z}}^{3} \subset \mathcal{H}_{g, 1}[[\infty]]$.

We also have the following important problem. It is not known that $\mathcal{H}_{g, 1}[[\infty]]$ coincides with the group

$$
\begin{aligned}
\mathcal{H}_{g, 1}[\infty] & :=\bigcap_{k} \operatorname{Ker}\left(\sigma_{k}: \mathcal{H}_{g, 1} \rightarrow \operatorname{Aut} N_{k}\right) \\
& =\operatorname{Ker}\left(\sigma^{\text {nil. }}: \mathcal{H}_{g, 1} \rightarrow \operatorname{Aut} F_{2 g}^{\text {nil }}\right),
\end{aligned}
$$

in which $\mathcal{H}_{g, 1}[[\infty]]$ is contained. This problem highly relates to the question whether $p: F_{2 g}^{\text {acy }} \rightarrow F_{2 g}^{\text {nil }}$ is injective or not.

\section{Refinements of the Johnson homomorphisms}

Finally, we summarize invariants of homology cylinders which extend the Johnson homomorphisms. For each $k \geq 2$, The $(k-1)^{\text {st }}$ Johnson homomorphism for homology cylinders is nothing other than the composite $\left.J_{k} \circ \sigma^{\text {acy }}\right|_{\mathcal{H}_{g, 1}[k]}$, where $\mathcal{H}_{g, 1}[k]:=$ $\operatorname{Ker}\left(\sigma_{k}: \mathcal{H}_{g, 1} \rightarrow \operatorname{Aut}_{0} N_{k}\right)$, and we now denote it by $J_{k}$, for short. We can determine the image of $J_{k}$ by using Theorem 2.3 due to Garoufalidis-Levine and Habegger. See [3] for details.

As seen in Section 5, we have a refinement

$$
\tilde{J}_{k}: \operatorname{Aut} F_{2 g}^{\text {acy }}[k] \rightarrow \operatorname{Hom}\left(F_{2 g},\left(\Gamma^{k} F_{2 g}\right) /\left(\Gamma^{2 k-1} F_{2 g}\right)\right)
$$

of the Johnson homomorphism $J_{k}$, so that we obtain an exact sequence

$$
1 \longrightarrow \mathcal{H}_{g, 1}[2 k-1] \longrightarrow \mathcal{H}_{g, 1}[k] \stackrel{\widetilde{J}_{k} \circ \sigma^{\text {acy }}}{\longrightarrow} \operatorname{Hom}\left(F_{2 g},\left(\Gamma^{k} F_{2 g}\right) /\left(\Gamma^{2 k-1} F_{2 g}\right)\right) \text {. }
$$

On the other hand, we can construct maps which are essentially the same as $\widetilde{J}_{k} \circ \sigma^{\text {acy }}$ by the following geometric method or group-homological one. This argument is based on Heap's idea in [6] for the case of the mapping class group.

The first one is obtained by applying the construction of the invariant $\theta$ in the previous section to the case of $\mathcal{H}_{g, 1}[k]$. The same argument gives a homomorphism $\theta_{k}: \mathcal{H}_{g, 1}[k] \rightarrow H_{3}\left(N_{k}\right)$ defined by

$$
\theta_{k}\left(\left[M, i_{+}, i_{-}\right]\right):=\widehat{f}\left(\left[C_{M}\right]\right) \quad \in H_{3}\left(N_{k}\right),
$$

and we can show that it is onto. Note that such a construction already appeared in [3]. 
Theorem 7.1 The kernel of $\theta_{k}$ is $\mathcal{H}_{g, 1}[2 k-1]$, namely we have an exact sequence

$$
1 \longrightarrow \mathcal{H}_{g, 1}[2 k-1] \longrightarrow \mathcal{H}_{g, 1}[k] \stackrel{\theta_{k}}{\longrightarrow} H_{3}\left(N_{k}\right) \longrightarrow 1 .
$$

Proof In [8], Igusa-Orr showed that the homomorphism $H_{3}\left(N_{2 k-1}\right) \rightarrow H_{3}\left(N_{k}\right)$ induced by the natural projection $N_{2 k-1} \rightarrow N_{k}$ is trivial. From this, we see that $\mathcal{H}_{g, 1}$ [2k$1] \subset \operatorname{Ker} \theta_{k}$. On the other hand, the induced homomorphism $\overline{\theta_{k}}: \mathcal{H}_{g, 1}[k] / \mathcal{H}_{g, 1}[2 k-$ 1] $\rightarrow H_{3}\left(N_{k}\right)$ turns out to be a surjective one between free abelian groups of the same rank (see [3] and [8]), which shows that $\overline{\theta_{k}}$ is an isomorphism. In particular, $\mathcal{H}_{g, 1}[2 k-1]=\operatorname{Ker} \theta_{k}$ follows.

The second one is obtained by generalizing Morita's refinement of the Johnson homomorphism for the mapping class group in [15] under the following modification. For $M \in \mathcal{H}_{g, 1}[k]$, we write $\varphi_{M}:=\sigma^{\text {acy }}(M) \in \operatorname{Aut}_{0} F_{2 g}^{\text {acy }}$. Let $C_{*}\left(F_{2 g}^{\text {acy }}\right)$ be the chain complex of $F_{2 g}^{\text {acy }}$ called the normalized standard resolution in [1]. We take a 2-chain $c_{0} \in C_{2}\left(F_{2 g}^{\text {acy }}\right)$ satisfying $\partial c_{0}=-(\zeta)$ and whose image in $C_{2}\left(\pi_{1} \Sigma_{g}\right)^{\text {acy }}$ by the map from $\Sigma_{g, 1}$ to the closed surface $\Sigma_{g}$ coincides with the natural image of the fundamental cycle of $C_{2}\left(\pi_{1} \Sigma_{g}\right)$. For each $\varphi_{M}, c_{0}-\varphi_{M}\left(c_{0}\right)$ gives a 2-cycle of $F_{2 g}^{\text {acy }}$. Since $H_{2}\left(F_{2 g}^{\text {acy }}\right)=0$, there exists a 3-chain $c_{M} \in C_{3}\left(F_{2 g}^{\text {acy }}\right)$ satisfying $\partial c_{M}=c_{0}-\varphi_{M}\left(c_{0}\right)$. Now $M \in \mathcal{H}_{g, 1}[k]$, so that $\varphi_{M}$ acts on $N_{k}$ trivially. Hence if we write $\bar{c}_{M}$ for the image of $c_{M}$ in $C_{3}\left(N_{k}\right)$, we obtain a 3-cycle $\bar{c}_{M}$ in $C_{3}\left(N_{k}\right)$. We define $\tilde{\tau}_{k}(M):=\left[\bar{c}_{M}\right] \in H_{3}\left(N_{k}\right)$. A similar argument to one in [15] shows that $\tilde{\tau}_{k}$ is well-defined and gives a refinement of $J_{k}$, where we need to use Igusa-Orr's result mentioned above instead of $H_{3}\left(F_{2 g}\right)=0$.

The same statement as Theorem 7.1 holds for $\tilde{\tau}_{k}$. To show that $\operatorname{Ker} \tilde{\tau}_{k}=\mathcal{H}_{g, 1}[2 k-1]$, we can apply Yokomizo's argument [18], where the exact sequence

$$
\begin{aligned}
H_{3}\left(N_{k+1}\right) \rightarrow H_{3}\left(N_{k}\right) \rightarrow H_{1}\left(F_{2 g}\right) \otimes\left(\Gamma^{k} F_{2 g}\right) /\left(\Gamma^{k+1} F_{2 g}\right) \rightarrow \\
\left(\Gamma^{k+1} F_{2 g}\right) /\left(\Gamma^{k+2} F_{2 g}\right)
\end{aligned}
$$

and Igusa-Orr's result are effectively used. The surjectivity of $\tilde{\tau}_{k}$ follows from Theorem 2.3 and a direct sum decomposition of $H_{3}\left(N_{k}\right)$ due to Igusa-Orr.

\section{References}

[1] KS Brown, Cohomology of groups, Graduate Texts in Mathematics 87, Springer, New York (1982) MR672956

[2] P M Cohn, Free rings and their relations, London Mathematical Society Monographs 19, Academic Press, London (1985) MR800091 
[3] S Garoufalidis, J Levine, Tree-level invariants of three-manifolds, Massey products and the Johnson homomorphism, from: "Graphs and patterns in mathematics and theoretical physics”, Proc. Sympos. Pure Math. 73, Amer. Math. Soc., Providence, RI (2005) 173-203 MR2131016

[4] N Habegger, Milnor, Johnson, and tree level perturbative invariants, preprint

[5] K Habiro, Claspers and finite type invariants of links, Geom. Topol. 4 (2000) 1-83 MR1735632

[6] A Heap, Bordism invariants of the mapping class group, preprint

[7] J Hillman, Algebraic invariants of links, Series on Knots and Everything 32, World Scientific Publishing Co., River Edge, NJ (2002) MR1932169

[8] K Igusa, K E Orr, Links, pictures and the homology of nilpotent groups, Topology 40 (2001) 1125-1166 MR1867241

[9] N Kawazumi, Cohomological Aspects of Magnus expansions, preprint

[10] P Kirk, C Livingston, Z Wang, The Gassner representation for string links, Commun. Contemp. Math. 3 (2001) 87-136 MR1820015

[11] J-Y Le Dimet, Enlacements d'intervalles et représentation de Gassner, Comment. Math. Helv. 67 (1992) 306-315 MR1161287

[12] J P Levine, Link concordance and algebraic closure. II, Invent. Math. 96 (1989) 571592 MR996555

[13] J P Levine, Algebraic closure of groups, from: "Combinatorial group theory (College Park, MD, 1988)", Contemp. Math. 109, Amer. Math. Soc., Providence, RI (1990) 99-105 MR1076380

[14] J Levine, Homology cylinders: an enlargement of the mapping class group, Algebr. Geom. Topol. 1 (2001) 243-270 MR1823501

[15] S Morita, Abelian quotients of subgroups of the mapping class group of surfaces, Duke Math. J. 70 (1993) 699-726 MR1224104

[16] K E Orr, Homotopy invariants of links, Invent. Math. 95 (1989) 379-394 MR974908

[17] J Stallings, Homology and central series of groups, J. Algebra 2 (1965) 170-181 MR0175956

[18] Y Yokomizo, private communication

Graduate School of Mathematical Sciences, the University of Tokyo

3-8-1 Komaba, Meguro-ku, Tokyo 153-8914, Japan

sakasai@ms.u-tokyo.ac.jp

Received: 13 October 2005 Revised: 10 February 2006 